\title{
Recent Advances in the Development of Laccase-Based Biosensors via Nano-Immobilization Techniques
}

\author{
Avinash A. Kadam ${ }^{1}\left(\right.$, Ganesh D. Saratale ${ }^{2}\left(\mathbb{D}\right.$, Gajanan S. Ghodake $^{3}{ }^{(0}$, Rijuta G. Saratale ${ }^{1}$, Asif Shahzad ${ }^{4}$, \\ Verjesh Kumar Magotra ${ }^{5}\left(\mathbb{D}\right.$, Manu Kumar ${ }^{6}\left(\mathbb{D}\right.$, Ramasubba Reddy Palem ${ }^{7}$ and Jung-Suk Sung ${ }^{6, *}(\mathbb{D})$
}

1 Research Institute of Biotechnology and Medical Converged Science, Dongguk University-Seoul, 32 Dongguk-ro, Ilsandong-gu, Goyang-si 10326, Gyeonggi-do, Korea; avikadam2010@gmail.com (A.A.K.); rijutaganesh@gmail.com (R.G.S.)

2 Department of Food Science and Biotechnology, Dongguk University-Seoul, 32 Dongguk-ro, Ilsandong-gu, Goyang-si 10326, Gyeonggi-do, Korea; gdsaratale@dongguk.edu

3 Department of Biological and Environmental Science, Dongguk University-Seoul, 32 Dongguk-ro, Ilsandong-gu, Goyang-si 10326, Gyeonggi-do, Korea; ghodakegs@gmail.com

4 Department of Energy and Materials Engineering, Dongguk University-Seoul, Seoul 04620, Korea; asifshzd8@gmail.com

5 Nano Information Technology Academy, Dongguk University, Jung-Gu, Seoul 100715, Korea; birju.srm@gmail.com

6 Department of Life Science, Dongguk University-Seoul, 32 Dongguk-ro, Ilsandong-gu, Goyang-si 10326, Gyeonggi-do, Korea; manukumar007@gmail.com

7 Department of Medical Biotechnology, Dongguk University-Seoul, 32 Dongguk-ro, Ilsandong-gu, Goyang-si 10326, Gyeonggi-do, Korea; palemsubbareddy@gmail.com

check for

updates

Citation: Kadam, A.A.; Saratale, G.D.; Ghodake, G.S.; Saratale, R.G.; Shahzad, A.; Magotra, V.K.; Kumar, M.; Palem, R.R.; Sung, J.-S. Recent Advances in the Development of Laccase-Based Biosensors via Nano-Immobilization Techniques. Chemosensors 2022, 10, 58 . https://doi.org/10.3390/ chemosensors 10020058

Academic Editor: Edelmira Valero

Received: 30 December 2021

Accepted: 28 January 2022

Published: 31 January 2022

Publisher's Note: MDPI stays neutral with regard to jurisdictional claims in published maps and institutional affiliations.

Copyright: (C) 2022 by the authors. Licensee MDPI, Basel, Switzerland. This article is an open access article distributed under the terms and conditions of the Creative Commons Attribution (CC BY) license (https:// creativecommons.org/licenses/by/ $4.0 /)$.
Abstract: Monitoring phenolic compounds is critical in the environmental, food, and medical sectors. Among many recent advanced detection platforms, laccase-based biosensing platforms gave very rapid, effective, online, and in situ sensing of phenolic compounds. In laccase-based biosensors, laccase immobilization techniques have a vital role. However, a detailing of the advancements in laccase immobilization techniques employed in laccase-based biosensors is lacking in the literature. Thus, in this review, we assessed how the nano-immobilization techniques shaped the laccase biosensing platforms. We discussed novel developments in laccase immobilization techniques such as entrapment, adsorption, cross-linking, and covalent over new nanocomposites in laccase biosensors. We made a comprehensive assessment based on the current literature for future perspectives of nano-immobilized laccase biosensors. We found the important key areas toward which future laccase biosensor research seems to be heading. These include 1. A focus on the development of multi-layer laccase over electrode surface, 2 . The need to utilize more covalent immobilization routes, as they change the laccase specificity toward phenolic compounds, 3 . The advancement in polymeric matrices with electroconductive properties, and 4 . novel entrapment techniques like biomineralization using laccase molecules. Thus, in this review, we provided a detailed account of immobilization in laccase biosensors and their feasibility in the future for the development of highly specific laccase biosensors in industrial, medicinal, food, and environmental applications.

Keywords: nano-immobilization; laccase; biosensor; emerging pollutants; monitoring; phenolic compounds

\section{Introduction}

A desire for a sophisticated life made humans resort to a vast number of synthetic compounds. Rapid fabrication advancements of these compounds caused an increase in hazardous, hardly-decomposable, and detrimental wastes [1]. The release of these synthetic compounds in natural water streams represents a substantial part of the emerging 
environmental contaminants (EECs) [1]. Worldwide, these EECs are entering the atmosphere very easily, on a daily basis and in increasing quantities. Phenolic compounds are a major part of these EECs, which accumulate, stay for a longer period in the environment, and eventually exert toxic effects on the ecosystem and humans [2]. The United States Environmental Protection Agency (USEPA) and the European Union (EU) declared phenolic compounds to be contaminants of priority concern [2]. Their major entry routes are different industrial, agricultural, and domestic processes. Most of these processes include the production of plastics, pharmaceuticals, petroleum refining, leather-based products, coking, coal conversion, textile dyes, pesticides, insecticides, paper/pulp manufacturing plants, etc. [1-4].

These hazardous phenolic compounds attracted great attention in the last decade due to their incomplete removal in wastewater treatment technologies [2], and hence they raised severe concerns about their adverse effect on human and environmental ecosystems [5]. Several phenolic compounds can trigger muscular weakness, damage to organs, serious corrosion, and damage to the central nervous system [6]. Additionally, phenolic compounds are also reported to be genotoxic and promote the formation of tumors $[5,7]$. Thus, because of these negative impacts, monitoring these phenolic compounds in natural waters, as well as those released in water after wastewater treatments, becomes obligatory [2]. The Environmental Protection Agency (EPA) set the maximum concentration of phenol at $0.006 \mu \mathrm{g} / \mathrm{L}$ for up to 10 days, which does not harm the environment [6,8]. Hence, the early monitoring of the phenolic compounds is very important, as the products of the secondary transformation reactions of these phenolic compounds under environmental conditions can be very detrimental to the ecosystem [2]. In addition to these environmental necessities, the need for monitoring the phenolic compounds from the food industry, pharmacological research, and biomedical analyses has also grown rapidly in recent times [9].

The techniques for the detection of phenolic compounds that are commonly used are spectrophotometry, high-performance liquid chromatography, gas chromatography, and capillary electrophoresis [10]. These well-reported techniques show major shortcomings, such as complex sample preparations, time-consuming procedures, and the demand for extremely expensive instruments. These shortcomings make on-site analysis unpractical, and the techniques cannot be applied in regular assessments. On the other hand, a biosensor is a tool that provides an easy, cost-effective, and rapid detection of phenolic compounds [11]. Therefore, there is a larger interest to develop low-cost, simple, small, and portable biosensors with good accuracy, sensitivity, rapidness, reproducibility, signal-tonoise ratio, resistance to the environment, long life, and operational safety [11-13].

Numerous biological components such as enzymes, living microorganisms, antigens, nucleic acids, antibodies, etc., can be utilized for the construction of biosensors. Among these biological components, microorganisms and enzymes are mainly utilized for biosensor construction $[11,14]$. The utilization of enzymatic biosensors increased significantly mainly due to important characteristics such as acting on specific substrates efficiently under milder conditions. These laid the base for the development of different enzymebased biosensors. The enzymatic biosensor employed for phenol sensing mainly involves oxidoreductive enzymes such as laccase, tyrosinase, lignin peroxidase, and horse-radish peroxidase [15]. Tyrosinase has limitations of low stability and product inhibition. Lignin peroxidase requires additional hydrogen peroxide to complete the reaction. This makes the biosensing platform complicated.

In contrast, laccase stands out as an excellent biosensor candidate for phenols, as it has i. a broad substrate specificity toward phenolic compounds, ii. the ability to catalyze the reaction without additional co-factors, and iii. the requirement of freely available molecular oxygen to carry out the reaction. Thus, the laccase biosensor reports were found to be increasing in recent times, with more and more advancements in laccase immobilization techniques (Figure 1). As laccases are an excellent candidate for phenol biosensing, a lot of advancements have been happening recently in laccase-based biosensors, with an improvement in the laccase immobilization techniques (Figure 1). However, a 
review focused on these advancements and the newest developments, particularly in the 'immobilization methods employed in laccase biosensors', is lacking. Because the laccase nano-immobilization techniques have a critical role in laccase-based biosensor application, the detailed evaluation of the comprehensive challenges posed by the nano-immobilization of laccases was not thoroughly reported.

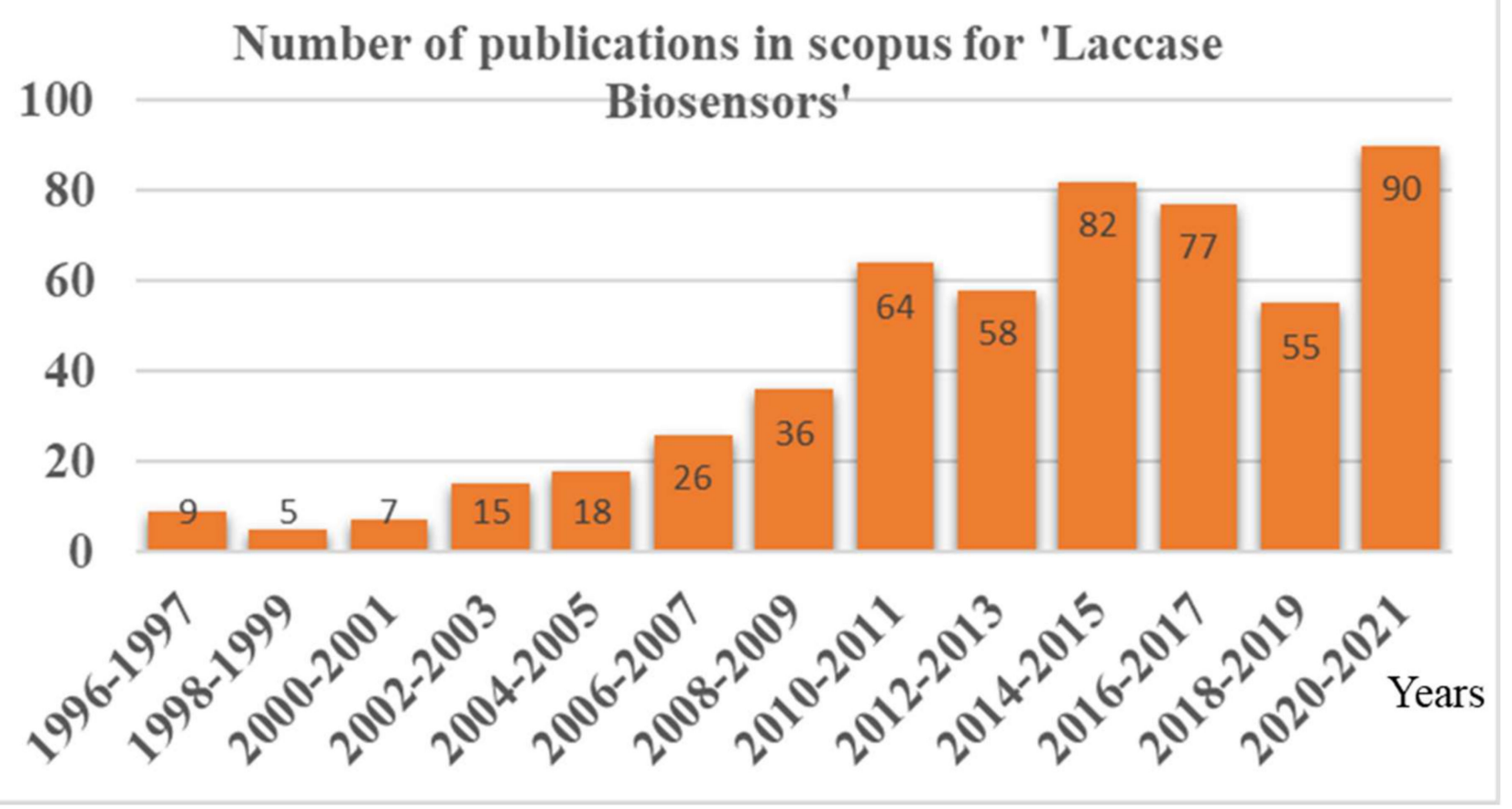

Figure 1. Year-wise publications in Scopus reported for the document search for 'Laccase Biosensors'.

Thus, the present review explains the recent advancements in laccase-based biosensors via novel nanomaterial-mediated laccase immobilization technologies. At first, the basics of laccase and laccase-based reactions for biosensing are briefly discussed. Afterwards, the detailed knowledge of the progression in the immobilization techniques and the need for laccase-based biosensors is critically analyzed. In the end, current challenges and constraints regarding the prospects for improving such laccase-based biosensors are reviewed.

\section{Laccase Information and Reaction}

Before looking into the biosensing application of laccase, it is essential to understand laccase's basic reaction, active site, and potential sources. The rationale of the laccase-based electrochemical/optical/thermal detection of phenolic compounds lies in the mechanism of the reaction catalyzed by laccase. Laccase belongs to a multi-copper-oxidase family of enzymes. Laccase (polyphenol oxidase; EC 1.10.3.2) catalytically oxidizes different organic and inorganic compounds, such as methoxy-substituted phenols, polyphenols, ketones, ascorbate, lignins, phosphates, and diamines [16-19]. Laccase catalyzes a series of typical reactions, in which substrates are oxidized through electron transfer to the molecular oxygen. The molecular oxygen is further reduced to the $\mathrm{H}_{2} \mathrm{O}$ molecule, with the complete oxidation of the substrate compounds $[20,21]$. Laccase's reaction mechanisms, active site structures, and the different sources of the laccase enzyme are explained in detail in Figure 2. 


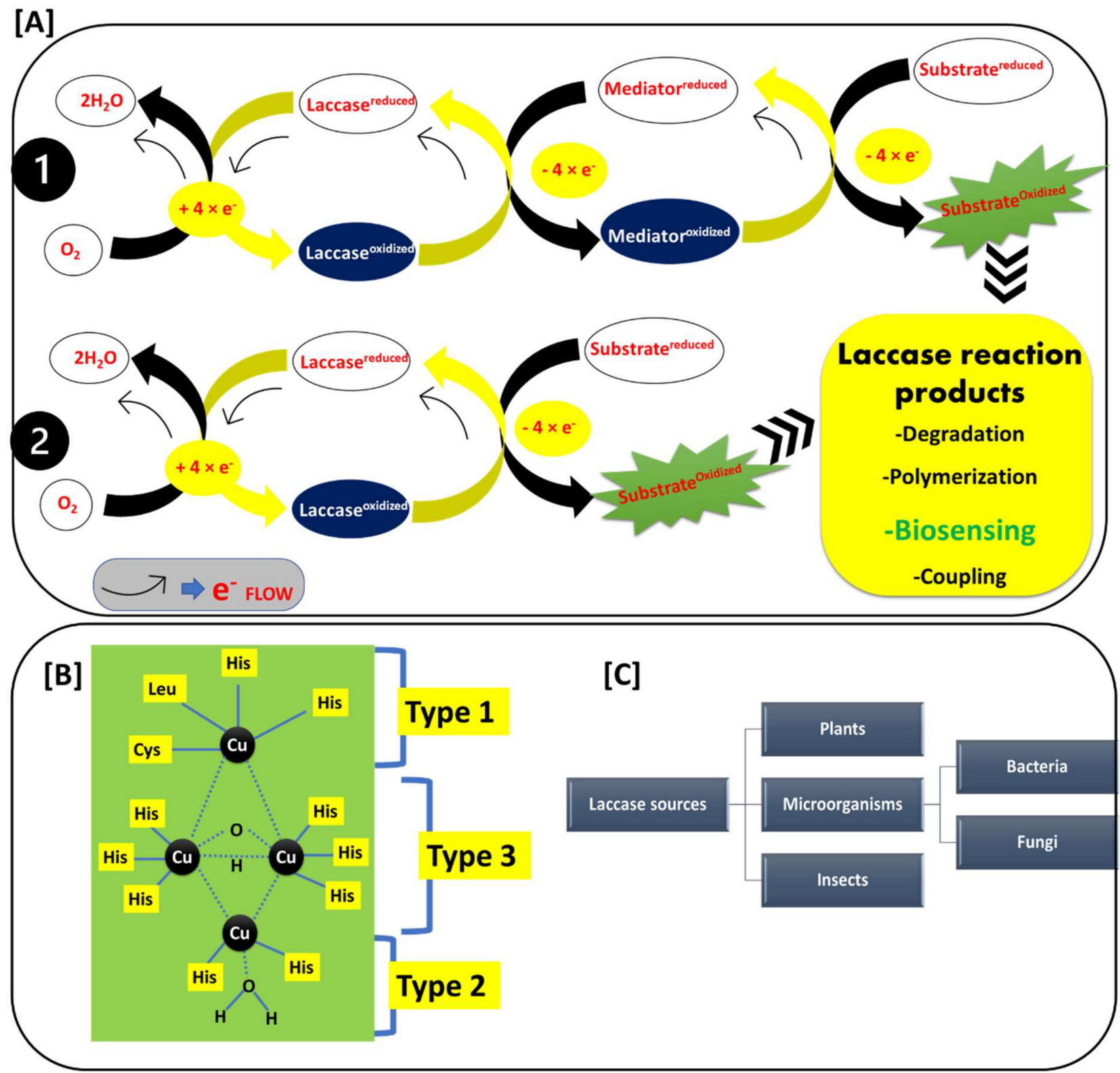

Figure 2. (A) Reaction mechanism of the laccase-catalyzed reactions: 1 . mediator dependent and 2. mediator independent reactions, and laccase reaction products; (B) Structure of the laccase active site Type 1, Type 2, and Type 3; and (C) Occurrence of laccase among various sources.

Figure 2A shows the basic laccase reaction mechanisms. Laccase reactions mainly involve two types of reactions: first, direct substrate oxidation Figure 2(A1); and second, the redox-mediated oxidation of the substrate (Figure 2(A2)) [22-25]. This depends upon the ionization potential of the substrate. If the ionization potential of the substrate is less than the redox potential of the T1 active site of laccase, the substrate can be directly oxidized by the laccase. If the ionization potential of the substrate is higher than the redox potential of the $\mathrm{T} 1$ active site of laccase, then the substrate oxidation is mediated by the redox mediator. In this case, the redox mediator gets oxidized by laccase, and it further oxidizes the target substrate. Generally, the phenolic compounds have an ionization potential that is lower than that of the T1 active site of laccase, and hence they form a phenoxy radical after the oxidation by laccase. In the typical reaction of substrate oxidation, laccase withdraws the electrons from the substrate and transfers them to the molecular oxygen. The transfer of the four electrons from the substrate to molecular 
oxygen successfully causes substrate oxidation and a simultaneous reduction of molecular oxygen to two water molecules Figure 2A. This broad substrate oxidization potential capability of laccase mainly represents its critical role in biotechnological applications such as degradation of xenobiotic compounds, polymerization reactions, biosensing of the phenolic compounds, coupling reactions, etc. $[23,25,26]$. This makes laccase a widely used, green, and inexpensive biotechnological tool that can be employed in a diverse range of applications [25].

Figure 2B shows the active site structure of laccase, where the laccase-catalyzed reaction is carried out. The laccase active site mainly involves three copper-binding sites (Type 1 (T1), Type 2 (T2), and Type 3 (T3)) [27-29]. The T1 active site center exhibits an intense spectrophotometric absorption at wavelengths of $600-610 \mathrm{~nm}$. This is mainly due to the covalent bond between copper and cysteine. This property gave laccase the typical blue color. In the T1 center, the copper atom is connected to two histidine residues and two sulfurs from different sulfur-containing amino acids. The sulfur-containing amino acids are varied as per the source of the laccase. In bacterial laccase, these sulfur-containing amino acids include methionine and cysteine, while the fungal laccase has leucine and phenylalanine [28]. The T1 center possesses a high redox potential, and hence it is the main site where the oxidation of the many phenolic substrates (which have lower redox potential than the T1 center) takes place. The T2 active site center is not detectable in a spectrophotometer, but it shows a strong electron paramagnetic resonance (EPR) signal. The copper atom in the T2 center is coordinated with the two histidine residues. The T3 center is binuclear, contains two copper atoms (connected by anti-ferromagnetic force), and each copper atom is connected to the three histidine residues, respectively. The T3 center has a characteristic spectrophotometric absorption at $330 \mathrm{~nm}$. This information on active sites helps us to understand the structural diversity of the laccases, and how laccases differentiate among different species [28].

Figure $2 \mathrm{C}$ shows the different sources of laccase. Laccase is widely reported in plants, micro-organisms, and insects [30-34]. In micro-organisms, bacterial and fungal laccase are widely studied sources and applied in many laccase-based applications [31]. Therefore, laccase is a ubiquitous enzyme with a broad occurrence in diverse species. Laccase's ability to oxidize the phenolic compounds set off lots of opportunities in the food, environmental, industrial, agricultural, and medical sectors. Thus, the application of laccase in the biosensing of phenols is a cheap and economical option.

\section{Structure of Typical Laccase-Based Biosensors}

The biosensor is defined as an analytical device in which the biological system (e.g., enzymes, receptors, antibodies, microorganisms, or cells) meets the sample analyte and transforms it into either an electric signal, an optical signal, a thermal signal, or another kind of signal [15,35-39]. The amplitude of the generated signal is directly related to the concentration of analytes in the solution. We schematically illustrated the typical structure of the laccase-based electrochemical and optical biosensors (Figure 3A,B). In the typical electrochemical biosensor, laccase immobilized on the sensor platform catalyzes the oxidation of the phenolic substrate. The oxidized phenolic substrate is further reduced by the electrons from the electrode. This completes the electrical cycle and produces the detectable electrochemical signal. Depending upon the measurement of this electrochemical signal by the detector system, electrochemical laccase biosensors are divided into amperometric, voltammetric, conductometric, and potentiometric biosensors [9]. 


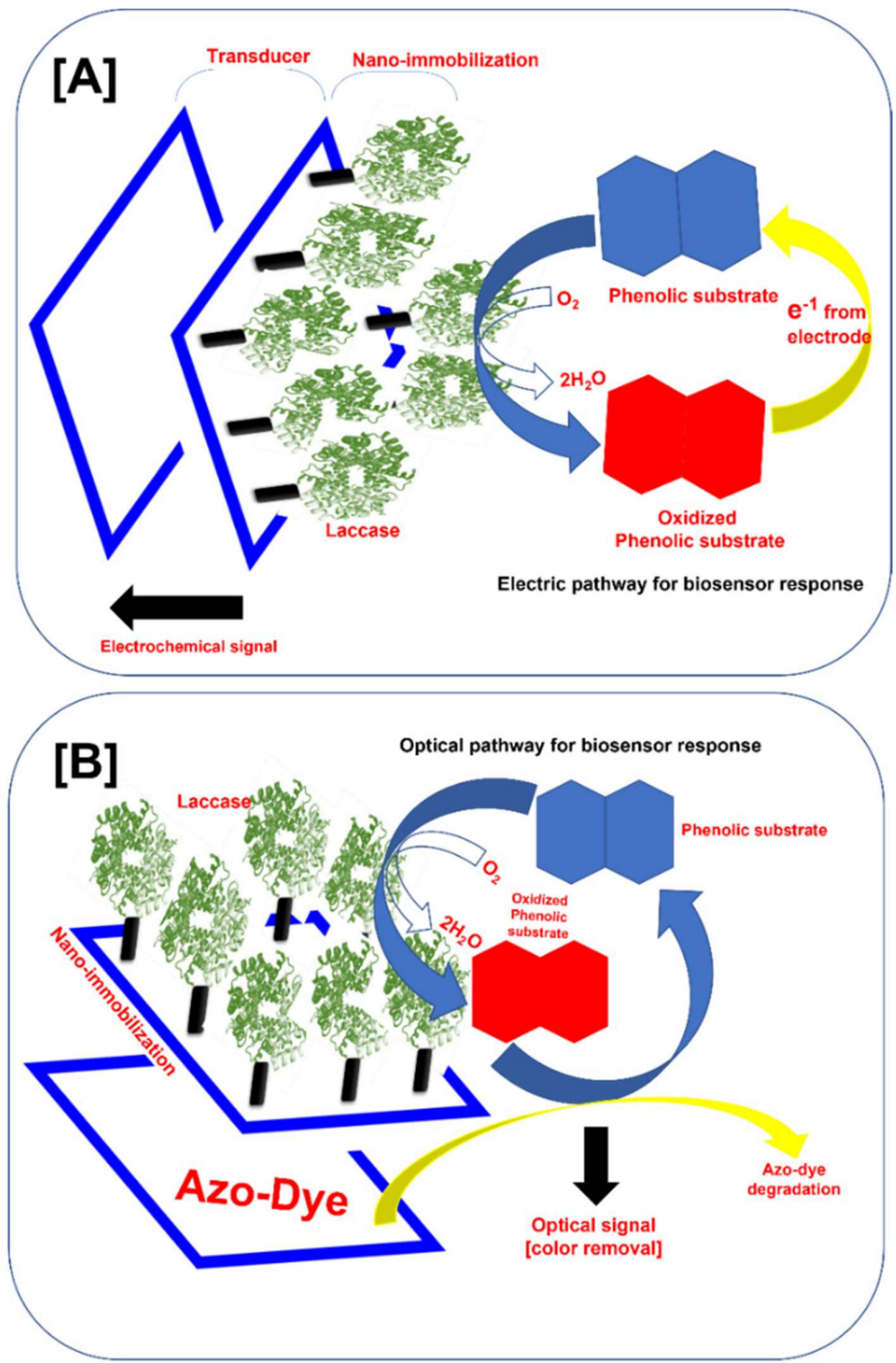

Figure 3. Typical structure of the nano-immobilized laccase-based biosensor for phenolic compound monitoring (A) Electrochemical sensor, and (B) optical sensor.

On the other hand, optical laccase-based biosensors measure the optical signal. Typically, the laccase immobilized on the biosensor platform oxidizes the phenolic compound, and the oxidized phenolic compound oxidizes the azo dye bound to the biosensor (Figure 3B) and gets reduced. The color change produces the optical signal directly related to the phenolic compound concentration [10]. This signal is further measured by the color change. In the optical biosensor, the response is very easy to detect and better for the on-site monitoring of the phenolic compound. In this biosensor, the use of organic 
nano-supports does not hamper the signal produced by the biosensor [10]. Nevertheless, the electro-chemical laccase-based biosensors can detect very low concentrations of phenolic compounds.

Furthermore, in the thermal laccase-based biosensors, the thermal response was measured. In these biosensors, after the laccase-catalyzed reaction, the reaction system's enthalpy change was measured [40]. Very few reports were available for thermal-based laccase biosensors. This presents the opportunity for major researchers in this field to investigate this aspect of laccase biosensors. The reviews by Rodríguez-Delgado et al., 2015, Sohrabi et al., 2017, and Castrovilli et al., 2019, explained this differentiation of the laccase biosensors in detail, citing the most important reports $[9,14,41]$. For all these different kinds of laccase-based biosensors, the immobilization of laccase plays a critical role. Thus, in this review, we focused on technological advancements in very recently developed nano-supports for laccase immobilization in laccase based-biosensors for phenolic compound detection.

\section{Nano-Immobilization of Laccase in Laccase-Based Biosensors}

On the road to applying laccase for biosensors, the main key lies in the immobilization of laccase on suitable supports. We schematically presented the basic differences in the reaction process of free laccase and immobilized laccase in Figure 4A. The applications of free laccase have many limitations. These include a one-time reaction, the difficulty to separate laccase and its products, and fewer stabilities due to a lack of support $(\mathrm{pH}$, operational temperatures, and storage stabilities). Thus, the application of laccase in free form is not practical. Consequently, laccase immobilizations play a key role. The immobilization of laccase mainly allows laccase to bound over a suitable support. This allows immobilized laccase to separate from the products, and it can be further applied in multiple cycles (Figure 4A). In addition to the multiple cycle use and the separation from the reaction's products, immobilization also brings enhanced stability to $\mathrm{pH}$, operational temperatures, and storage times. This ultimately improves the operational range of the immobilized laccase in biosensors [9]. In the laccase immobilization process, the nature of the supports has great importance. The immobilization supports for laccase immobilization were divided into reversible and irreversible depending upon the reaction between laccase and the nature of the supports. We presented this classification of the immobilization supports schematically in Figure 4B. In the irreversible immobilization process, laccase is immobilized on the support with either covalent bonding, entrapments, or cross-linking, while in the reversible immobilizations laccase was immobilized on the various supports through adsorption and disulfide linkage (Figure 4B). Earlier, the immobilization of laccase was focused on conventional supports [34]. These supports include alginate beads, polymeric resins, and biopolymer matrix [39]. These supports have shown laccase immobilization; however, they have major limitations for the laccase-based biosensors. These limitations include a smaller available surface area to immobilize laccase and a lower fit in portable miniature biosensor platforms. Moreover, due to its bulky/organic nature, it hampers the electrochemical/optical properties as response measurements. Hence, the construction of the laccase biosensor using conventional immobilization supports becomes very difficult [9].

A solution to this problem was found in the advancement of nano-support platforms. Recent advances in nanotechnology offered an array of nanomaterials with extremely organized sizes, shapes, and interesting properties. Nanomaterials reveal huge possibilities in many fields of science, including biosensor development [41]. In the last decade, an enormous advancement was evident in the development of highly efficient nanoformulations for the immobilization of laccase [42]. These nanomaterials broadly include carbon-based nanomaterials (graphene oxide, carbon nanotubes, and carbon dots (CDs), etc.), metal-oxide nanomaterials $\left(\mathrm{Fe}_{3} \mathrm{O}_{4}, \mathrm{Fe}_{2} \mathrm{O}_{3}, \mathrm{CuO}, \mathrm{AuNPs}, \mathrm{AgNPs}, \mathrm{ZnO}\right.$, titania, zirconia, and alumina, etc.), clay-based nanomaterials (halloysite, kaolinite, laponite, sepiolite, bentonite, and 
montmorillonite, etc.), and nanobiocomposites (fabricated with crafting biopolymers and nanomaterials) [30-33].

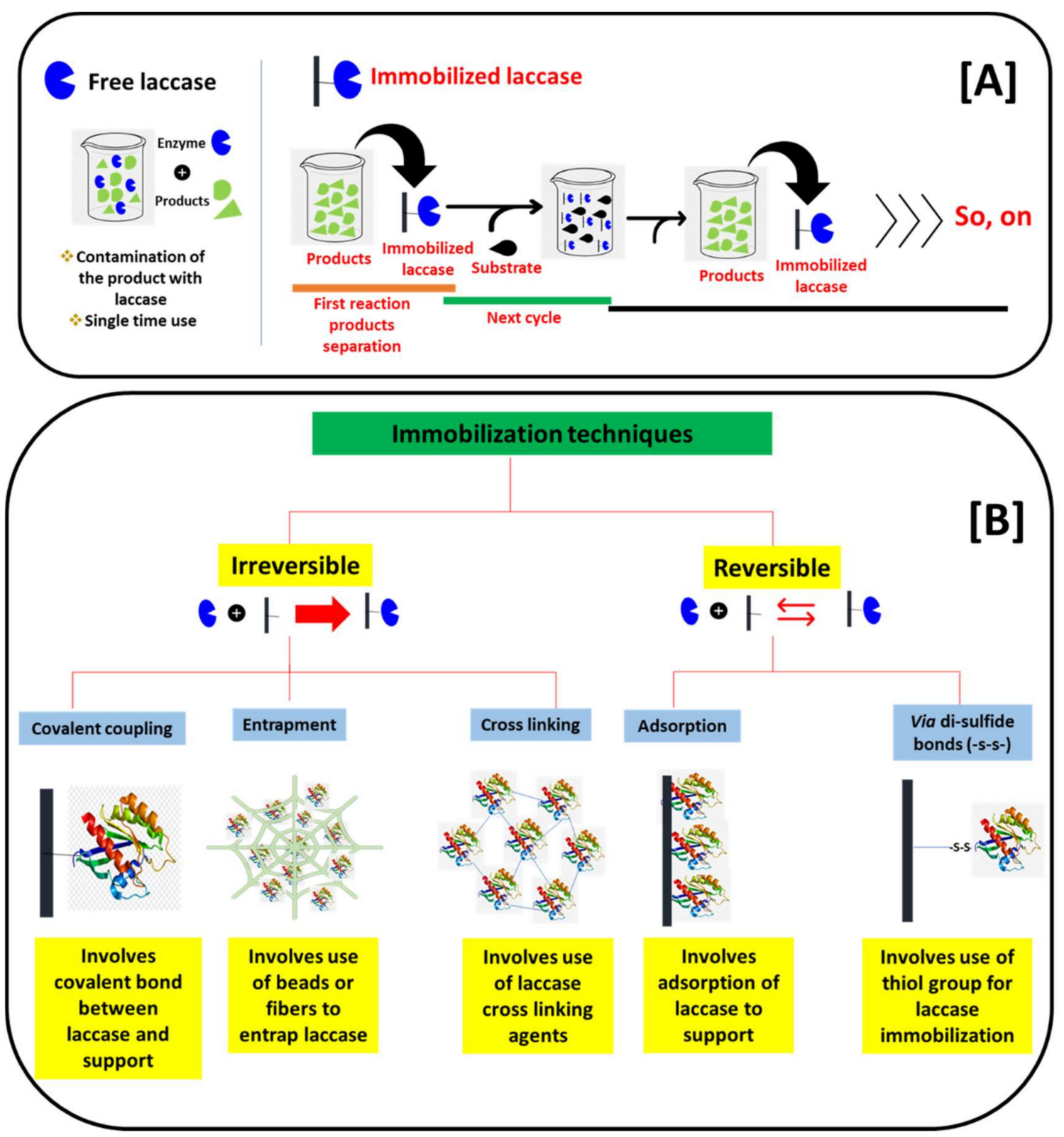

Figure 4. Schematic presentation of the (A) basic difference between free and immobilized laccasecatalyzed reactions, and the $(\mathbf{B})$ differentiation of laccase immobilization techniques based on a binding pattern with a support material.

The major advantages of these nano-supports for laccase immobilizations were highlighted in Figure 5. They include the incorporation of the highly specific properties and surface advancements that nanoscale materials can offer for enzyme immobilizations [43]. These include magnetic, optical, electrical, chemical, and anti-bacterial properties [39]. The introduction of this magnetic property in the biosensor not only improves the sensitivity but also offers a controllable biomolecule immobilization method. Remarkably, due to the use of magnetic properties in immobilized laccase biosensors, we can utilize an external magnetic field that can control electrocatalytic and electrocatalytic processes [44]. The magnetic properties in nano-supports allow for an easy separation of the nano-supports from the reaction mixture, and the magnetically immobilized laccase can be utilized in multiple cy- 
cles for the desired applications [45-50]. In laccase biosensors, along with laccase-catalyzed oxidation reactions, the optical and electrochemical properties offered by immobilization nano-supports are highly important in eliciting the sensor performance [9,30]. Moreover, the anti-bacterial properties increase the shelf life of the sensors. In addition to these properties, nanomaterials also increase the surface-to-volume ratio for the immobilization of laccase to occur [42]. This automatically increases the immobilization capacity of laccase. An increase in the surface-to-volume ratio also adds to an increase in enzyme-substrate interaction [43]. This leads to the increased and effective catalysis of the enzymes. Furthermore, nanocomposites provide multi-point attachment sites on the same support. This increases the binding capacity of the laccase to the supports. With solid multi-point binding, the chances of getting released into the reaction mixtures reduce significantly. The multipoint attachments also support the enzyme for the enhancement of the catalytic potential with increased $\mathrm{pH}$, thermal, and storage stabilities [43]. Thus, to apply laccase for phenol biosensing, laccase immobilization on a robust nano-support with specific properties (optical, electrical, or magnetic), a high surface to volume ratio, and multi-point attachments are crucial.

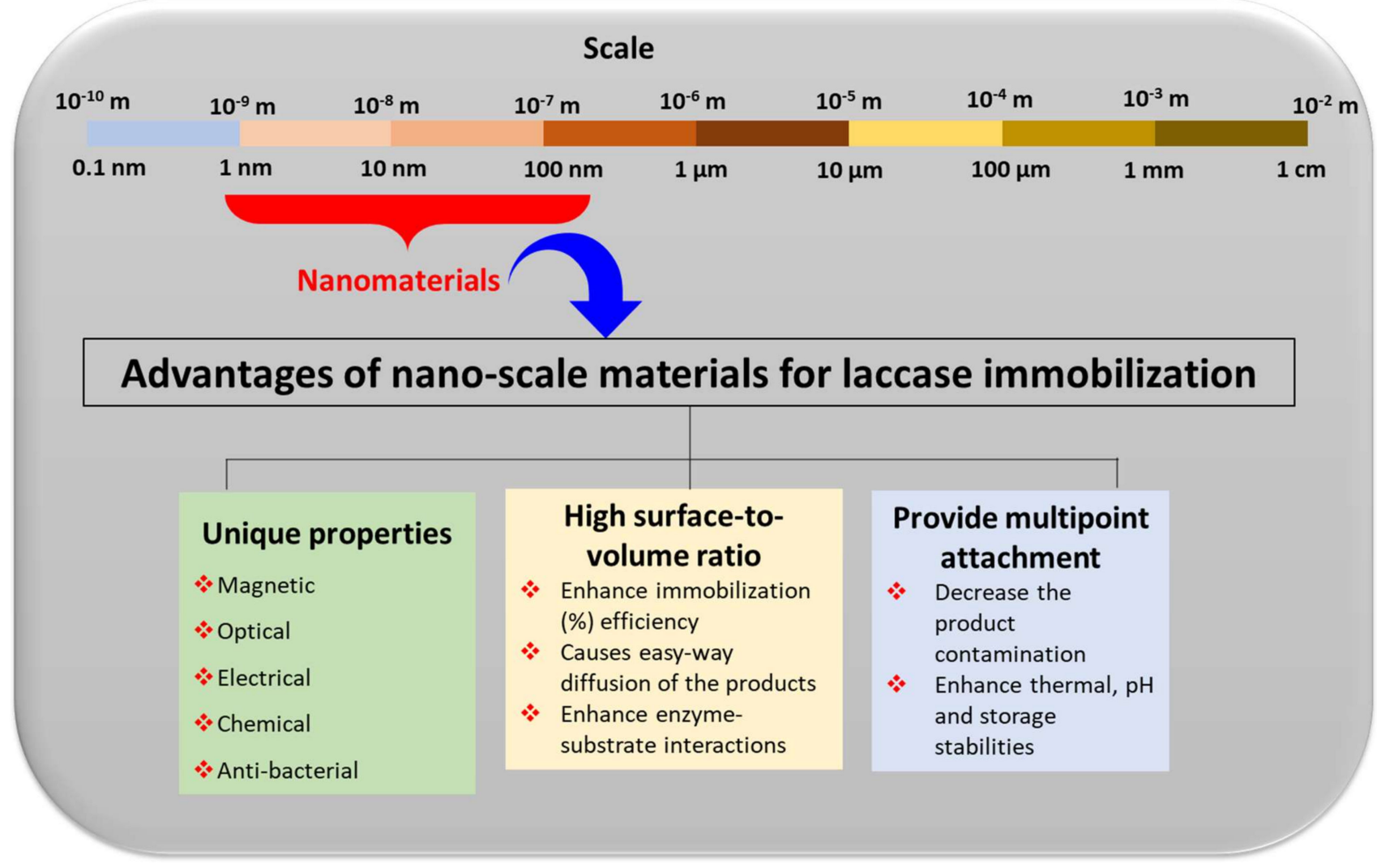

Figure 5. Schematic presentation of the advantages of the nanoscale materials for the laccase immobilization process.

The comprehensive overview of recently reported advanced nano-immobilization supports in laccase-based biosensors is evaluated in detail in Table 1. This evaluation includes the type of nano-immobilization support, a modification made on the nanosupport, the cross-linking agent, the laccase source, the characteristics of the laccase, the immobilization conditions, and the main immobilization type. Along with this, Table 1 also details biosensor parameters such as the phenolic compound detected, the range of the phenolic compound evaluated, the transducing method of the signal measurements, the sensitivity of the developed biosensor with real sample analysis, the stability of the laccase in a biosensor, and the application in the specific field. 
Table 1. The details of nano-immobilization processes and biosensor parameters analyzed in recently developed laccase-based biosensors.

\begin{tabular}{|c|c|c|c|c|c|c|c|c|c|c|}
\hline \multicolumn{5}{|c|}{ Nano-Immobilization Parameters } & \multicolumn{5}{|c|}{ Biosensor Development } & \multirow[b]{2}{*}{ Ref } \\
\hline Nano-Supports & $\begin{array}{l}\text { Modification } \\
\text { Made with } \\
\text { Nano-Support }\end{array}$ & Laccase Source & $\begin{array}{l}\text { Immobilization } \\
\text { Conditions ( } \mathrm{pH}: \mathrm{P}, \\
\left.\text { Temperature: } \mathrm{T}^{\circ} \mathrm{C}\right)\end{array}$ & $\begin{array}{c}\text { Type of } \\
\text { Immobilization }\end{array}$ & $\begin{array}{l}\text { Analyte (Analyte } \\
\text { Detection Range) }\end{array}$ & $\begin{array}{c}\text { Biosensor } \\
\text { Type/Transduction }\end{array}$ & $\begin{array}{l}\text { Stability of Laccase } \\
\text { in Biosensor }\end{array}$ & $\begin{array}{l}\text { Selectivity/Real } \\
\text { Samples }\end{array}$ & Application & \\
\hline $\begin{array}{c}\text { Carboxyl } \\
\text { functionalized } \\
\text { multi-walled carbon } \\
\text { nanotubes } \\
\text { (COOH-MWCNT) }\end{array}$ & PEI coated AuNP & $\begin{array}{l}\text { Coriolus hirsuta } \\
\text { (Trametes hirsuta) }\end{array}$ & $\begin{array}{l}\mathrm{P}: 4.2, \mathrm{~T} \text { : room } \\
\text { temperature }\end{array}$ & Adsorption & $\begin{array}{l}\text { Catechol// } \\
(0-1 \mathrm{mM})\end{array}$ & $\begin{array}{l}\text { Electrochemical/ } \\
\text { Amperometric }\end{array}$ & $\begin{array}{l}77 \% \text { catalytic response } \\
\text { in } 10 \text { cycles. Laccase } \\
\text { retained } 86.0 \% \text { after } \\
30 \text { days }\end{array}$ & - & Environment & [51] \\
\hline Silica nanoparticles & Phytic acid & Trametes versicolor & - & Adsorption & $\begin{array}{c}\text { Dopamine } \\
(0.99-103.10 \mu \mathrm{M})\end{array}$ & $\begin{array}{l}\text { Electrochemical/ } \\
\text { Amperometric }\end{array}$ & $\begin{array}{c}\text { After } 20 \text { days of } \\
\text { storage at } 4{ }^{\circ} \mathrm{C}(>90 \%)\end{array}$ & $\begin{array}{l}\text { Determination of the } \\
\text { recovery of DA in } \\
\text { pharmaceutical } \\
\text { injection }\end{array}$ & $\begin{array}{l}\text { Pharmacological } \\
\text { research }\end{array}$ & [52] \\
\hline $\begin{array}{l}\text { Graphene-cellulose } \\
\text { Microfiber }\end{array}$ & - & Trametes versicolor & - & Adsorption & $\begin{array}{c}\text { Catechol/ } \\
(0.2 \text { to } 209.7 \mu \mathrm{M})\end{array}$ & $\begin{array}{l}\text { Electrochemical/ } \\
\text { Voltammetric }\end{array}$ & $\begin{array}{l}\text { After } 132 \mathrm{~h} 96.8 \% \\
\text { initial response } \\
\text { retained }\end{array}$ & $\begin{array}{l}\text { Detection of catechol } \\
\text { in different } \\
\text { water samples }\end{array}$ & Environment & [53] \\
\hline $\begin{array}{c}\mathrm{Cu}_{3}\left(\mathrm{PO}_{4}\right)_{2} \cdot 3 \mathrm{H}_{2} \mathrm{O} \\
\text { microflowers }\end{array}$ & - & Trametes versicolor & P: $7.4, \mathrm{~T}: 4$ & Entrapment & $\begin{array}{c}\text { Epinephrine } \\
(0.4-400 \mu \mathrm{g} / \mathrm{mL})\end{array}$ & $\begin{array}{l}\text { Optical/ } \\
\text { Colorimetric }\end{array}$ & $\begin{array}{l}\text { Laccase reserved } 96.6 \% \\
\text { and } 64.4 \% \text { of initial } \\
\text { activity after stored for } \\
30 \text { days and } 5 \text { recycles }\end{array}$ & $\begin{array}{c}\text { Favorable } \\
\text { applicability for EP } \\
\text { detection in human } \\
\text { blood serum and } \\
\text { urine samples }\end{array}$ & $\begin{array}{l}\text { Clinical diagnosis } \\
\text { and } \\
\text { pharmacological } \\
\text { research. }\end{array}$ & [54] \\
\hline $\begin{array}{c}\text { Multiwalled } \\
\text { carboxylic- } \\
\text { functionalized } \\
\text { carbon nanotubes }\end{array}$ & $\begin{array}{l}\text { Chitosan solution, } \\
\text { BMIMBF }_{4} \text { ionic } \\
\text { liquid }\end{array}$ & Trametes versicolor & $\mathrm{T}: 4$ & Entrapment & $\begin{array}{l}\text { Bisphenol A/ } \\
(0.5-12 \mu \mathrm{M})\end{array}$ & $\begin{array}{l}\text { Electrochemical/ } \\
\text { Potentiostat- } \\
\text { galvanostat }\end{array}$ & $\begin{array}{l}\text { Laccase kept } 87 \% \text { of } \\
\text { the initial response } \\
\text { after one month }\end{array}$ & $\begin{array}{l}\text { Bisphenol A detection } \\
\text { from river water }\end{array}$ & Environment & [55] \\
\hline $\begin{array}{c}\mathrm{Cu}_{3}\left(\mathrm{PO}_{4}\right)_{2} \cdot 3 \mathrm{H}_{2} \mathrm{O} \\
\text { microflowers }\end{array}$ & - & Trametes versicolor & P: $7.4, \mathrm{~T}: 4$ & Entrapment & $\begin{array}{l}\text { Epinephrine/ } \\
(0.4-400 \mu \mathrm{M})\end{array}$ & $\begin{array}{l}\text { Optical/ } \\
\text { Colorimetric }\end{array}$ & $\begin{array}{l}\text { Laccase decreased the } \\
\text { sensing capacity of } \\
\text { epinephrine only } 2 \% \\
\text { after being stored for } \\
30 \text { days }\end{array}$ & $\begin{array}{l}\text { Ascorbic acid, citric } \\
\text { acid, vitamin C, } \\
\text { glucose, glycine, } \\
\text { L-lysine, and urea } \\
\text { had no interference } \\
\end{array}$ & $\begin{array}{c}\text { Clinical diagnosis } \\
\text { and } \\
\text { pharmacological } \\
\text { research }\end{array}$ & [56] \\
\hline AuNPs/SPCE & Polypyrrole & - & P: 7, T: 4 & Entrapment & $\begin{array}{l}\text { Polyphenols/ } \\
(1-250 \mu \mathrm{M})\end{array}$ & $\begin{array}{l}\text { Electrochemical/ } \\
\text { Amperometric }\end{array}$ & $\begin{array}{l}\text { Laccase sensor showed } \\
85 \% \text { activity retention } \\
\text { after one-month } \\
\text { storage }\end{array}$ & $\begin{array}{l}\text { Polyphenols in } \\
\text { propolis samples }\end{array}$ & Food & [57] \\
\hline $\begin{array}{l}\text { Poly (glycidyl } \\
\text { methacrylate-co-n } \\
\text { butyl acrylate) } \\
\text { microspheres }\end{array}$ & $\begin{array}{l}\text { Colloidal AuNPs, } \\
\text { Glutaraldehyde }\end{array}$ & - & - & Entrapment & $\begin{array}{l}\text { Tartrazine } / \\
(0.2 \text { to } 14 \mu \mathrm{M})\end{array}$ & $\begin{array}{l}\text { Electrochemical/ } \\
\text { Amperometric }\end{array}$ & $\begin{array}{l}\text { Laccase biosensor } \\
\text { response was stable up } \\
\text { to } 30 \text { days }\end{array}$ & $\begin{array}{l}\text { Foods and beverages } \\
\text { used as real samples }\end{array}$ & Food & [19] \\
\hline
\end{tabular}


Table 1. Cont.

\begin{tabular}{|c|c|c|c|c|c|c|c|c|c|c|}
\hline \multicolumn{5}{|c|}{ Nano-Immobilization Parameters } & \multicolumn{5}{|c|}{ Biosensor Development } & \multirow[b]{2}{*}{ Ref } \\
\hline Nano-Supports & $\begin{array}{l}\text { Modification } \\
\text { Made with } \\
\text { Nano-Support }\end{array}$ & Laccase Source & $\begin{array}{c}\text { Immobilization } \\
\text { Conditions ( } \mathrm{pH}: \mathrm{P} \\
\left.\text { Temperature: } \mathrm{T}^{\circ} \mathrm{C}\right)\end{array}$ & $\begin{array}{c}\text { Type of } \\
\text { Immobilization }\end{array}$ & $\begin{array}{l}\text { Analyte (Analyte } \\
\text { Detection Range) }\end{array}$ & $\begin{array}{c}\text { Biosensor } \\
\text { Type/Transduction }\end{array}$ & $\begin{array}{l}\text { Stability of Laccase } \\
\text { in Biosensor }\end{array}$ & $\begin{array}{l}\text { Selectivity/Real } \\
\text { Samples }\end{array}$ & Application & \\
\hline Carbon nanofiber & $\begin{array}{l}\text { Magnetic, } \\
\text { Polydopamine } \\
\text { (PDA), nickel } \\
\text { nanoparticle }\end{array}$ & Trametes versicolor & P: 5.5 & Entrapment & $\begin{array}{c}\text { Catechol/ } \\
\text { (1 } \mu \mathrm{M} \text { to } 9.1 \mathrm{mM})\end{array}$ & $\begin{array}{l}\text { Electrochemical/ } \\
\text { Amperometric }\end{array}$ & $\begin{array}{l}\text { Laccase-biosensor } \\
\text { response to catechol } \\
\text { was within } 2.0 \% \text { for } \\
10 \text { successive } \\
\text { measurements } \\
\text { indicated good } \\
\text { stability. }\end{array}$ & $\begin{array}{c}\text { Catechin, epicatechin, } \\
\text { gallic acid, guaiacol, } \\
\text { phenol, and } \\
\text { aminophenol had no } \\
\text { interference/catechol } \\
\text { successfully detected } \\
\text { in real tap water, and } \\
\text { lake water }\end{array}$ & Environment & [44] \\
\hline $\mathrm{TiO}_{2} / \mathrm{CuCNFs}$ & Nafion polymer & Trametes versicolor & P: 6.8 & Entrapment & $\begin{array}{l}\text { Hydroquinon/ } \\
(1-89.8 \mu \mathrm{M})\end{array}$ & $\begin{array}{l}\text { Electrochemical/ } \\
\text { Chronoamperometry }\end{array}$ & $\begin{array}{l}\text { Even after a month, the } \\
\text { biosensor still retained } \\
93.45 \% \text { of the } \\
\text { initial response }\end{array}$ & $\begin{array}{l}\text { No interference from } \\
\text { guaiacol, 3,5-dinitro } \\
\text { salicylic acid, vanillin, } \\
\text { phenol, and catechol }\end{array}$ & Environment & [58] \\
\hline $\begin{array}{c}\text { Platinum } \\
\text { nanoparticles and } \\
\text { reduced graphene } \\
\text { oxide }\end{array}$ & Nafion polymer & Trametes versicolor & - & Entrapment & $\begin{array}{l}\text { Caffeic acid/ } \\
(0.2-2 \mu \mathrm{M})\end{array}$ & $\begin{array}{l}\text { Electrochemical/ } \\
\text { Amperometric }\end{array}$ & $\begin{array}{l}\text { After six weeks, a } \\
\text { remnant response of } \\
87.4 \% \text { from its } \\
\text { initial value }\end{array}$ & $\begin{array}{l}\text { Total polyphenolic } \\
\text { content from } \\
\text { tea infusions }\end{array}$ & Food & [59] \\
\hline AuNPs-MoS 2 & Nafion polymer & - & P: 5, T: 4 & Entrapment & $\begin{array}{c}\text { Catechol } \\
(2-2000 \mu \mathrm{M})\end{array}$ & $\begin{array}{l}\text { Electrochemical/ } \\
\text { Amperometric }\end{array}$ & $\begin{array}{c}\text { Ten repeated cycles } \\
\text { gave a } 3 \% \text { decreased } \\
\text { response }\end{array}$ & $\begin{array}{l}\text { Resorcinol, salicylic } \\
\text { acid, phenol, and } \\
\text { p-nitrophenol do not } \\
\text { affect the response }\end{array}$ & Environment & [60] \\
\hline $\begin{array}{l}\text { Microporous carbon } \\
\text { fibers }\end{array}$ & $\begin{array}{l}\text { Cathodic polymer } \\
\text { GY 83-0270 } 0005\end{array}$ & Trametes versicolor & P: 4.5 & Entrapment & Catechol, ABTS & $\begin{array}{l}\text { Electrochemical/ } \\
\text { Amperometric }\end{array}$ & - & Wastewater sample & Environment & [62] \\
\hline $\begin{array}{l}\text { Pyrene-terminated } \\
\text { block copolymer }\end{array}$ & $\begin{array}{l}\text { Pyrolytic graphite } \\
\text { (HOPG) }\end{array}$ & $\begin{array}{l}\text { Trametes } \\
\text { Versicolor }\end{array}$ & $\mathrm{T}: 4$ & Entrapment & $\begin{array}{l}\text { Pyrocatechol/ } \\
(50 \mathrm{nM} \text { to } 1 \mathrm{Mm})\end{array}$ & $\begin{array}{l}\text { Electrochemical/ } \\
\text { Voltammetric }\end{array}$ & $\begin{array}{c}\text { After } 30 \text { days at } 4{ }^{\circ} \mathrm{C} \\
\text { response decreased } \\
\text { by } 4.23 \%\end{array}$ & $\begin{array}{c}\text { Highly sensitive } \\
\text { (lowest detection limit } \\
\text { of } 50 \mathrm{nM} \text { ) }\end{array}$ & Environment & [63] \\
\hline
\end{tabular}


Table 1. Cont.

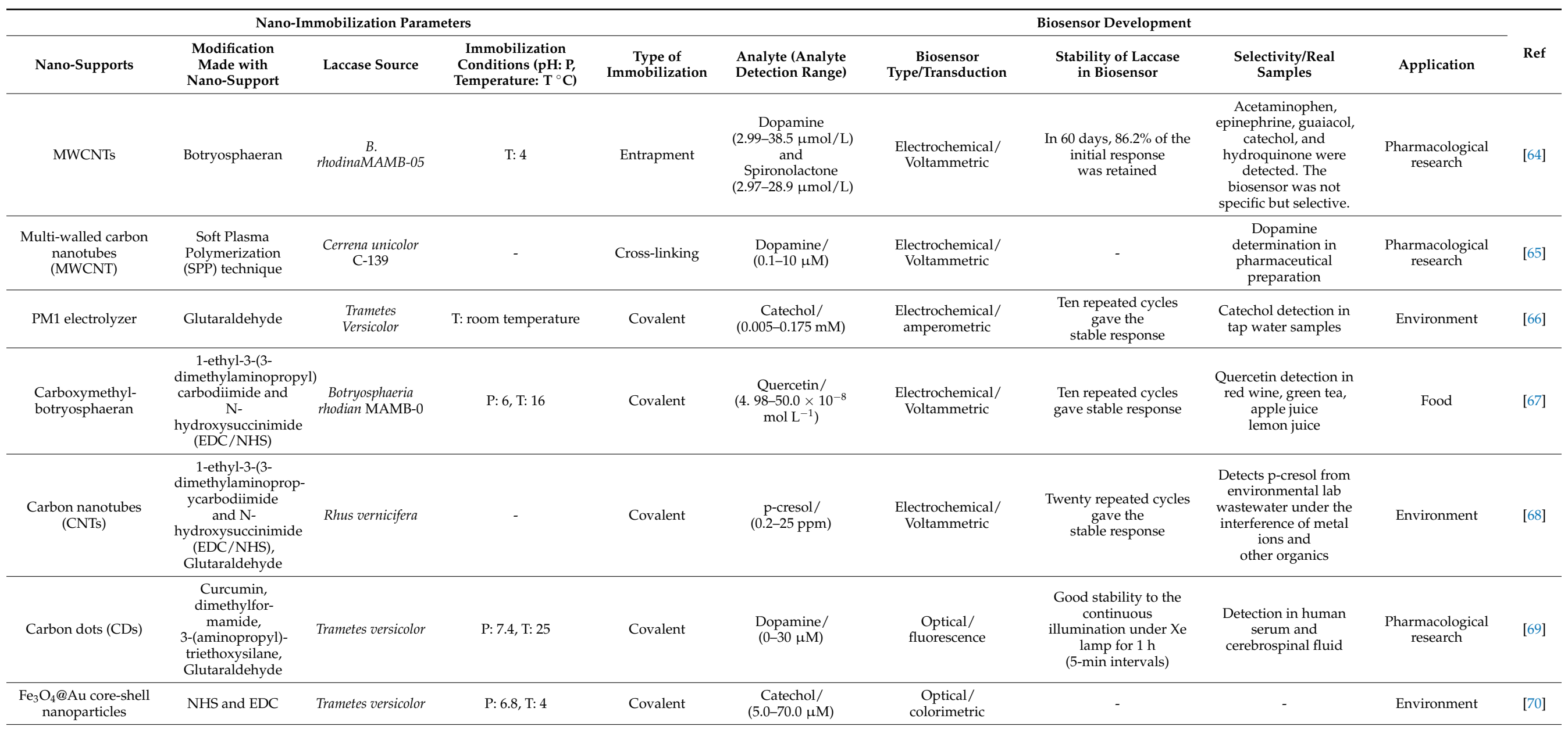


Table 1. Cont.

\begin{tabular}{|c|c|c|c|c|c|c|c|c|c|c|}
\hline \multicolumn{5}{|c|}{ Nano-Immobilization Parameters } & \multicolumn{5}{|c|}{ Biosensor Development } & \multirow[b]{2}{*}{ Ref } \\
\hline Nano-Supports & $\begin{array}{l}\text { Modification } \\
\text { Made with } \\
\text { Nano-Support }\end{array}$ & Laccase Source & $\begin{array}{c}\text { Immobilization } \\
\text { Conditions }(\mathrm{pH}: \mathrm{P}, \\
\left.\text { Temperature: } \mathrm{T}^{\circ} \mathrm{C}\right)\end{array}$ & $\begin{array}{c}\text { Type of } \\
\text { Immobilization }\end{array}$ & $\begin{array}{l}\text { Analyte (Analyte } \\
\text { Detection Range) }\end{array}$ & $\begin{array}{c}\text { Biosensor } \\
\text { Type/Transduction }\end{array}$ & $\begin{array}{l}\text { Stability of Laccase } \\
\text { in Biosensor }\end{array}$ & $\begin{array}{l}\text { Selectivity/Real } \\
\text { Samples }\end{array}$ & Application & \\
\hline $\begin{array}{l}\mathrm{Fe}_{2} \mathrm{O}_{3} \text { yolk-shell } \\
\text { particle }\end{array}$ & $\begin{array}{l}\text { Glutaraldehyde, } \\
\text { APTES, } \\
\text { carbodiimide, } \\
\text { cyano, and PEI }\end{array}$ & $\begin{array}{l}\text { Trametes } \\
\text { Versicolor }\end{array}$ & - & Covalent & $\begin{array}{c}\text { 2,6- } \\
\text { dimethoxyphenol } \\
(0.25-250 \mu \mathrm{M})\end{array}$ & $\begin{array}{l}\text { Electrochemical/ } \\
\text { Voltammetric }\end{array}$ & - & $\begin{array}{l}\text { Gave a response to } \\
\text { other phenolic } \\
\text { compounds, as well } \\
\text { as guaiacol, } \\
\text { pyrogallol, and } \\
\text { L-dopa/synthetic } \\
\text { wastewater }\end{array}$ & Environment & [71] \\
\hline $\begin{array}{l}\text { Plasma-polymerized } \\
\text { allylamine (PPAA) }\end{array}$ & $\begin{array}{l}\text { EDC/NHS } \\
\text { activation }\end{array}$ & $\begin{array}{l}\text { Trametes versicolor } \\
\text { (ATCC 32745) }\end{array}$ & P: 7 & Covalent & $\begin{array}{c}2,6- \\
\text { dimethoxyphenol } \\
\text { (DMP) }\end{array}$ & $\begin{array}{l}\text { Electrochemical/ } \\
\text { Amperometric }\end{array}$ & - & $\begin{array}{l}\text { Biosensor retained } \\
\text { laccase activity for } \\
\text { more than } 6 \text { months }\end{array}$ & Environment & [38] \\
\hline $\begin{array}{l}\text { Gold nanoparticles } \\
\text { (AuNPs) }\end{array}$ & $\begin{array}{l}\text { Poly-L-arginine, } \\
\text { Glutaraldehyde }\end{array}$ & $\begin{array}{l}\text { Trametes } \\
\text { Versicolor }\end{array}$ & P: 6 & Covalent & $\begin{array}{l}\text { Catechol// } \\
\text { (24-274 nM) }\end{array}$ & $\begin{array}{l}\text { Electrochemical/ } \\
\text { Voltammetric }\end{array}$ & - & $\begin{array}{l}\text { Catechin, phenol, and } \\
\text { aminophenol did not } \\
\text { affect sensitivity/tap } \\
\text { and river water }\end{array}$ & Environment & [73] \\
\hline Carbon dots (CDs) & $\begin{array}{c}\text { PFTBD, } \\
\text { Glutaraldehyde }\end{array}$ & Trametes versicolor & P: 7 & Covalent & $\begin{array}{c}\text { Catechol// } \\
(1.25-175 \mu \mathrm{M})\end{array}$ & $\begin{array}{l}\text { Electrochemical/ } \\
\text { Amperometric }\end{array}$ & $\begin{array}{c}\text { Ten repeated cycles } \\
\text { gave a stable response }\end{array}$ & $\begin{array}{l}\text { Detection of catechol } \\
\text { in tap water }\end{array}$ & Environment & [74] \\
\hline Carbon Nanofibers & $\begin{array}{l}\text { Cobalt } \\
\text { phthalocyanine, } \\
\text { Glutaraldehyde }\end{array}$ & $\begin{array}{l}\text { Trametes } \\
\text { Versicolor }\end{array}$ & - & Covalent & $\begin{array}{c}\text { p-Coumaric Acid/ } \\
(0.4-6.4 \mu \mathrm{M})\end{array}$ & $\begin{array}{l}\text { Electrochemical/ } \\
\text { Voltamperometric }\end{array}$ & $\begin{array}{l}\text { Biosensor laccase } \\
\text { decreases } 10.56 \text { and } \\
10.20 \% \text { after } 8 \text { days }\end{array}$ & $\begin{array}{l}\text { Gallic acid, ascorbic } \\
\text { acid, vanillic acid, and } \\
\text { ferrulic acid have not } \\
\text { shown much } \\
\text { interference }\end{array}$ & Environment & [76] \\
\hline Graphene oxide & $\begin{array}{l}\text { Carbodiimide } \\
\text { (EDAC), } \\
\text { Sulfo-NHS } \\
\text { solution }\end{array}$ & Agaricus bisporus & - & Covalent & $\begin{array}{c}\text { Catechol/ } \\
(0-1.6 \mathrm{mmol} / \mathrm{L})\end{array}$ & $\begin{array}{l}\text { Electrochemical/ } \\
\text { Voltammetric }\end{array}$ & - & $\begin{array}{l}\text { EDTA and benzoic } \\
\text { acid inhibited } \\
\text { laccase reaction }\end{array}$ & Environment & [77] \\
\hline Chitosan/AuNPs & $\begin{array}{l}\text { Phthalocyanine, } \\
\text { Glutaraldehyde }\end{array}$ & Agaricus bisporus & P: 7 & Covalent & $\begin{array}{c}\text { Catechol/ } \\
(2.4-20.0 \mathrm{mmol} / \mathrm{L})\end{array}$ & $\begin{array}{l}\text { Electrochemical/ } \\
\text { Amperometric }\end{array}$ & $\begin{array}{c}\text { Good repeatability in } \\
3 \text { cycles }\end{array}$ & - & Environment & [78] \\
\hline
\end{tabular}


The survey (Table 1) of the recently reported nano-supports utilized for the immobilization of laccase in biosensors concluded that the major immobilization types are adsorption, entrapment, cross-linking, and covalent bonding. We further discuss detailed recent advancements in these immobilization types in the following subsections.

\subsection{Adsorption-Based Laccase Immobilization}

The adsorption of the laccase on nanostructured electrodes involves a loading of the laccase by means of mainly hydrophobic, hydrogen, and Van-der-Waals interactions between laccase and the nano-supports. Wang et al., 2014, reported the adsorptive immobilization of the laccase on phytic acid-modified $\mathrm{SiO}_{2}$ nanoparticles [52]. This study involves a very simple protocol, simply adding the laccase solution over the surface of the nanostructured $\left(\mathrm{SiO}_{2}\right)$ electrode surface [52]. The laccase immobilized on the graphenecellulose microfiber composite modified the screen-printed carbon electrode for a sensitive determination of catechol. Palanisamy et al., 2016 also involves the simple adsorption method [53]. Laccase adsorption on nanostructured electrodes is a simple and easy process. However, it lacks the permanent binding with the electrode surface, and hence it affected the biosensor performance and long-term consistencies [69]. On the contrary, Othman and Wollenberger, 2020 reported the immobilization of the laccase on MWCNT-COOH/SPCE via electrostatic adsorption, with a significantly higher biosensing ability than other immobilization techniques [51]. Othman and Wollenberger, 2020 immobilized laccase using various types of immobilization strategies, such as entrapment, cross-linking, and covalent methods, in addition to electrostatic adsorption [51]. Othman and Wollenberger, 2020 confirmed the higher performance of the electrostatic adsorption immobilization strategy and proposed the hypothesis that the obtained result was due to the preservation of the laccase structure by the adsorption method [51]. Thus, the effectiveness of the adsorption process depends upon the maintenance of laccase's structural integrity. The main advantage of the adsorption-based laccase immobilization process is the simple, rapid, and easy immobilization. However, the adsorption of laccase can be less stable at various conditions due to the lack of permeant binding to the support. This affects the efficacy of the sensor performance. Hence, in advanced laccase biosensors, the adsorption technique is less preferred.

\subsection{Entrapment-Based Laccase Immobilization}

According to recent reports, the entrapment approach was found to be the major type of immobilization in laccase biosensor development (Table 1). Among the entrapment techniques reported, biomineralization is a modern and extremely novel approach for the direct and cost-effective immobilization of laccase for laccase-based biosensors. Biomineralization is an excellent process, in which the fabrication of advanced hierarchical architectures encompassing minerals and enzyme frameworks in aqueous environments under ambient conditions is possible [54]. Zang et al., 2022 assembled $\mathrm{Cu}_{3}\left(\mathrm{PO}_{4}\right)_{2} \cdot 3 \mathrm{H}_{2} \mathrm{O}$ microflowers by coordination between copper ions of $\mathrm{Cu}_{3}\left(\mathrm{PO}_{4}\right)_{2} \cdot 3 \mathrm{H}_{2} \mathrm{O}$ crystals and amide groups of laccase molecules via the biomineralization process [54]. The typical biomineralization strategy for laccase immobilization and its further application detection of phenolic compound epinephrine is explained in Figure 6 [54]. The $\mathrm{Cu}$ ions directly co-ordinate with the amide group of the laccase and form the nanosheet monomers. Further, in the growth phase, they form the three-dimensional microflowers entrapped with the laccase (Figure 6). Zhang et al., 2022, also utilized this biomineralization process for the immobilization of laccase and achieved an excellent smartphone-based detection of epinephrine molecules from water [56]. The formed biominerals contain immobilized enzymes with unique biofunctionalities, comprising excellent mechanical and physicochemical properties in an external harsh environment. This gives additional benefits in the laccase-based biosensor applications [53,54]. Thus, biomineralization is the new and advanced entrapment immobilization approach that can be further developed for laccase immobilization and its application in the development of laccase-based biosensors. 


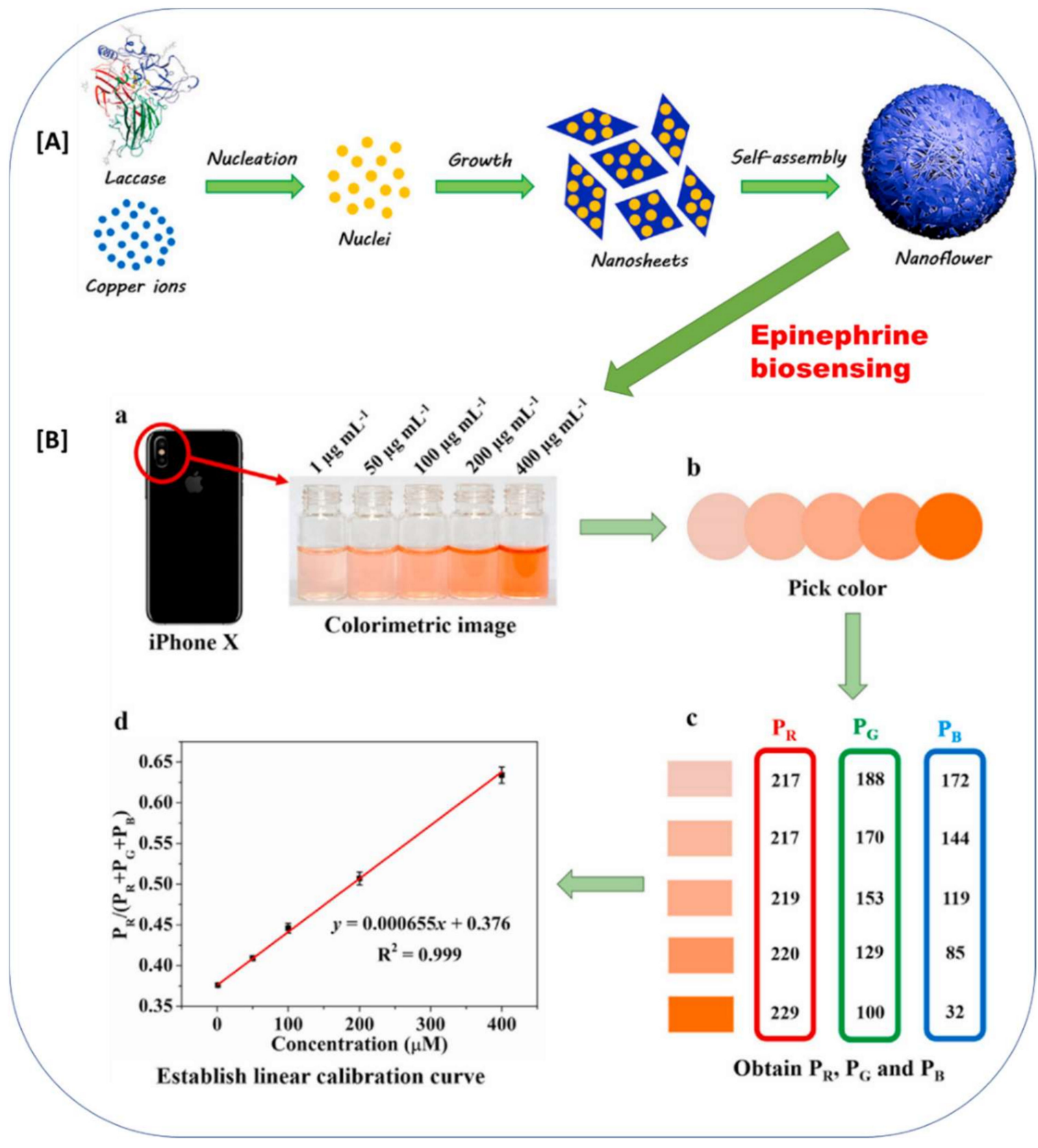

Figure 6. The biomineralization approach for laccase immobilizations in biosensing of the epinephrine. (A) Schematic representation of the formation of laccase-mineral $\left(\mathrm{Cu}_{3}\left(\mathrm{PO}_{4}\right)_{2} \cdot 3 \mathrm{H}_{2} \mathrm{O}\right)$ hybrid microflowers, (B) Application of laccase-mineral $\left(\mathrm{Cu}_{3}\left(\mathrm{PO}_{4}\right)_{2} \cdot 3 \mathrm{H}_{2} \mathrm{O}\right)$ hybrid microflowers for epinephrine biosensing; (a) Colorimetric image captured by iPhone $\mathrm{X}$ camera; (b) pick color via ColorPicker app; (c) obtain PR, PG, and PB; (d) establish a linear calibration curve. (The image (A) and (B) and respective figure captions were adapted from the Zhang et al., 2021 [56] with permission from Elsevier, Amsterdam, The Netherlands (License number: 5216550495018)).

Furthermore, Coelho et al., 2019 reported the fungal botryosphaeran coated on MWCNTs for the loading of the laccase [47]. The laccase was successfully entrapped in the fungal EPS and yielded a significant biosensing of dopamine and spironolactone [47]. Bravo et al., 2022 utilized a chitosan biopolymer for the entrapment of the laccase in the electrode formation using the multi-wall carbon nanotubes as a template support in the biosensor [55]. Despite the many advantages of chitosan as an entrapment agent, its poor electrochemical properties hampered the biosensor performance, as it resists the electron 
transfer process. Bravo et al., 2022 overcame this limitation of chitosan by incorporating highly conductive materials, such as electrically conductive carbon nanotubes [55]. The chitosan-entrapped laccase on multi-wall carbon nanotubes is effectively utilized for the comprehensive detection of the bisphenol-A. Thus, this proves that the use of organic immobilization agents requires the incorporation of the highly conductive nanomaterial to combat/overcome the electron transfer limitation in laccase-based biosensors.

The polymeric microsphere's structure remains an advantageous tool for the entrapment-based immobilization of enzymes due to its 3D shape with a high surface area, porosity, chemical stability, and density of the functional groups. These microspheres can easily be tailored in accord with their specific needs. Mazlan et al., 2017 developed the entrapment-based immobilization of laccase by using the functionalized methacrylateacrylate microspheres [19]. Poly (glycidyl methacrylate) (PGMA) encompasses an adequate number of epoxy groups, which have been widely applied for enzyme immobilization. The biosensor platform developed by Mazlan et al., 2017 is a combination of the gold nanoparticles (AuNPs) coated on a carbon-paste screen-printed electrode and laccaseconjugated microspheres [19]. Mazlan et al., 2017 applied this biosensor for the detection of tartrazine with significant laccase stability.

Furthermore, additional advancements in entrapment techniques were seen in recent years. These advancements include a combination of electrospinning, polymerization, and magnetized nano-support for laccase immobilization. Luo et al., 2014 developed the nano-supports by electro-spinning first, and later the biosensor was prepared by the polymerization of laccase on its surface with dopamine and a magnetic nanocomposite [44]. Thus, Luo et al., 2014 made an excellent magnetic biosensor platform, which can be easily stayed on the magnetic electrode surface. The magnetic property of the nano-support held it with the magnetic support. This technique eased the electrode preparation complications. The detailed schematic presentation of this advanced laccase-biosensor synthesis was presented by Luo et al., 2014 [44]. Electrospinning has been identified as an easy, lowcost, and powerful material for the manufacturing of many nanostructures for sensing applications, and these electrospun fibers are huge possibilities in the field of laccase-based biosensors [79].

Electropolymerization is another important laccase entrapment immobilization technique. Electropolymerization is a simple means of acquiring polymer films via the current that is applied to the electrode or regulating the number of cycles. This process was well studied and is one of the evolving methods of fabrication of sensor platforms. Amongst the numerous electrochemical systems, the combination of screen-printed carbon electrodes (SPCE) with amperometric detection is found to be one of the most suitable options [57]. The literature survey suggests that the electrochemical transducing methods got growing attention in laccase-based biosensors (Table 1). This is mainly because of their fast response, high sensitivity, low instrumental costs, simplicity, portability, and small sample volumes [41]. SPCE provides the most suitable platform for electrochemical laccase-based biosensors. Thus, combining this SPCE platform and electropolymerization holds high importance in electrochemical biosensor construction. The electropolymerization by polypyrrole for laccase entrapment on the SPCE platform was achieved by Mohtar et al., 2019 [57]. Polypyrrole is an electroactive and conductive polymer. Because of its simple deposition of dopant and entrapping ability of macromolecules onto biosensor electrode surfaces, polypyrrole is widely used in many biosensor platforms.

The typical schematic presentation of laccase entrapment onto the SPCE platform by polypyrrole-mediated electropolymerization and its application for the quantification of polyphenols in propolis samples are displayed in Figure 7. Mohtar et al., 2019 obtained excellent results for biosensing the polyphenols in a real-world sample of propolis [57]. Thus, the development of the combination of the SPCE platform and electropolymerization can be a suitable option for electrochemical laccase biosensor developments. 


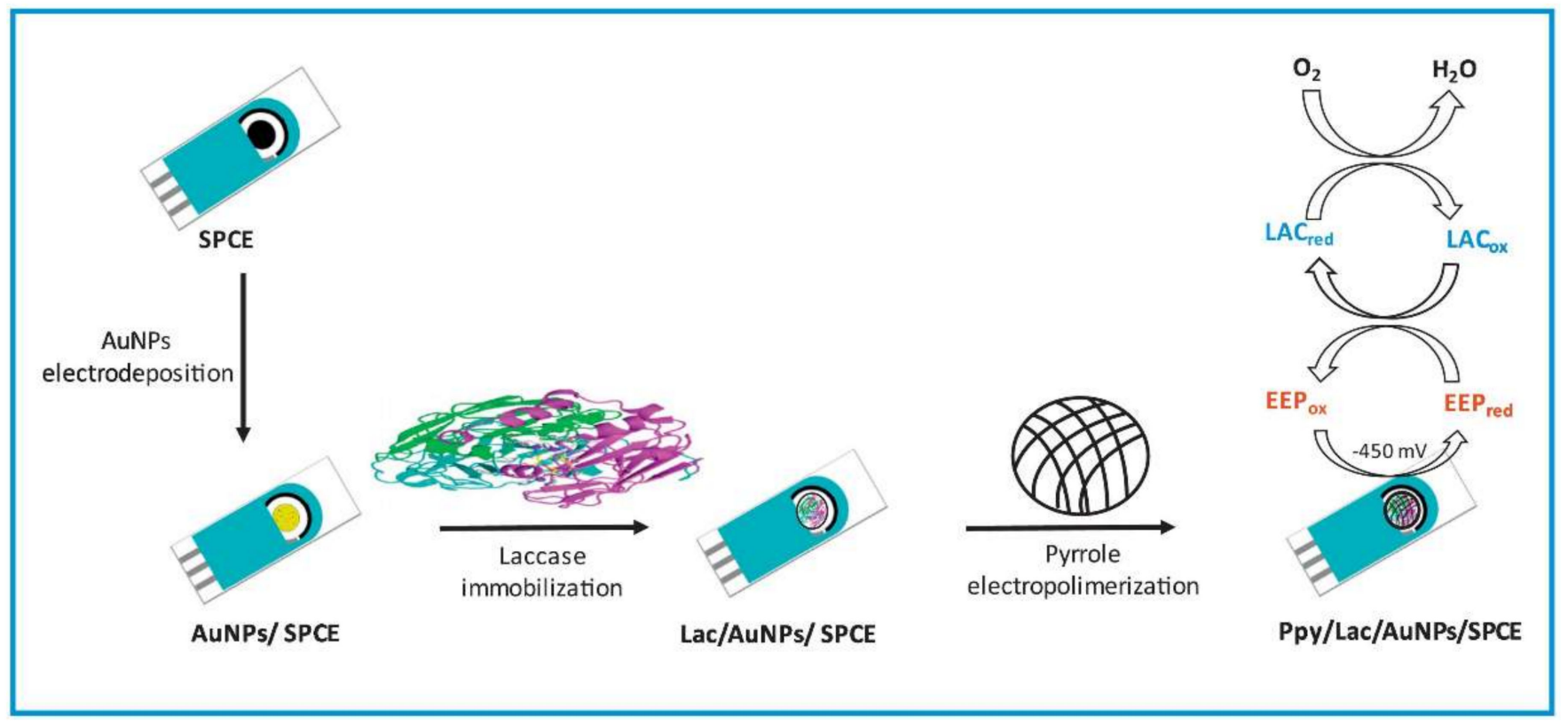

Figure 7. A schematic illustration of the screen-printed carbon electrodes (SPCE) modification with the Ppy/Lac/AuNPs nanocomposite film for the quantification of polyphenols in propolis samples (This image and its caption are adapted from Mohtar et al., 2019 [57] with permission from Elsevier, Amsterdam, The Netherlands (License number 5217650406979).

Another important process employed by many researchers for electrochemical laccasebased biosensors is the use of the Nafion polymer for laccase entrapment over the electrode surface $[58-61,80]$. Nafion is a synthetic polymer and a brand name of the sulfonated tetrafluoroethylene-based fluoropolymer-copolymer. Nafion is a powerful electrochemical binder and possesses an outstanding proton conductivity, excellent mechanical and thermal stabilities, and the capability of film formation, which is certainly valuable in immobilizing laccase on biosensor platforms [11,58]. Kavetskyy et al., 2020, used cathodic paint GY 83-0270 0005 to entrap a layer of laccase microporous carbon fibers. They developed electroconductive immobilization matrices using the laccase, microporous carbon fibers, and cathodic paint GY 83-0270 0005, and further studied the effect of their structure on operational parameters of laccase-based amperometric biosensors [62]. The cathodic paint GY 83-0270 0005 is mainly utilized for electrodeposition to form the biorecognition layer [81].

Moreover, Chen et al., 2017, developed a polymeric $\mathrm{Cu}^{2+} / \mathrm{PAA}$ (poly(acrylic acid))/ PPEGA (poly(poly-(ethylene glycol) acrylate)) matrix for the successful entrapment of laccase on graphene paper [63]. The $\mathrm{Cu}^{2+}$ PAA/PPEGA polymeric matrix entrapped the laccase via a $\pi$-conjugated graphene-like surface and $\pi-\pi$ stacking interactions between the pyrene moiety of PAA/PPEGA [63]. Chen et al., 2017 applied this biosensor for the stable and effective detection of the pyrocatechol [63].

The major limitation of this technique lies in the decrease in the functional laccase layer on the electrode, as most of the area is acquired by the entrapment polymer. Moreover, the entrapment technique cannot sure of the permeant binding of the laccase to the entrapment polymer. Hence, a loss of the laccase biomolecules in biosensor application can occur in subsequent cycles. The use of an organic/bio polymer is environmentally and economically sustainable, but ultimately affects the electron transduction process in electrochemical laccase biosensors. The use of these biopolymers for entrapment can be vital for the other sensing methods, such as the optical ones, considering its sustainability and economic feasibility. The use of electroconductive polymers for laccase entrapment provides an excellent advantage to electrochemical biosensor performance. The main advantage of the entrapment technique for laccase immobilization is entrapping catalytically active laccase 
in a simple, easy, and rapid way with a suitable polymer. Finally, our review suggests that various upcoming entrapment technologies, such as biomineralization, electron spinning techniques, magnetic entrapment polymers, electro polymerization, electroconductive polymers (Nafion and GY 83-0270 0005), and microspheres can be vital for the development of laccase-based biosensors. These methods gave the sense of projection and progression of the laccase biosensor development. Ultimately, looking at this novel entrapment immobilization technique, they can take over a major fraction of the upcoming laccase-based biosensors in the future.

\subsection{Cross-Linking-Based Laccase Immobilization}

The cross-linking immobilization technique involves the cross-linkages between the enzymes. Wardak et al., 2020 reported laccase-based biosensors by using the innovative, one-step, and eco-friendly Soft Plasma Polymerization (SPP) technique [65]. We schematically present the typical instrument construct of the SPP setup in Figure 8, from [65]. It involves components such as pointed tungsten electrodes, conductive solid support, grounded electrode, laccase enzyme solution, atomizer, and helium gas. This SPP technique employs the use of low-energy corona discharges of cold plasma with temperatures under $40{ }^{\circ} \mathrm{C}$ generated under atmospheric pressure. The discharge was produced in the pin-toplane structure in between the two tungsten needle electrodes in the presence of helium carrier gas. These ambient conditions of polymerization enable the direct incorporation of biomolecules like laccase in fibers for biosensing application [82]. The use of the SPP technique in the direct fabrication of biosensor recognition layers allows for an outstanding process simplification and a reduction in time for sensor fabrication from several hours to a few minutes [82].

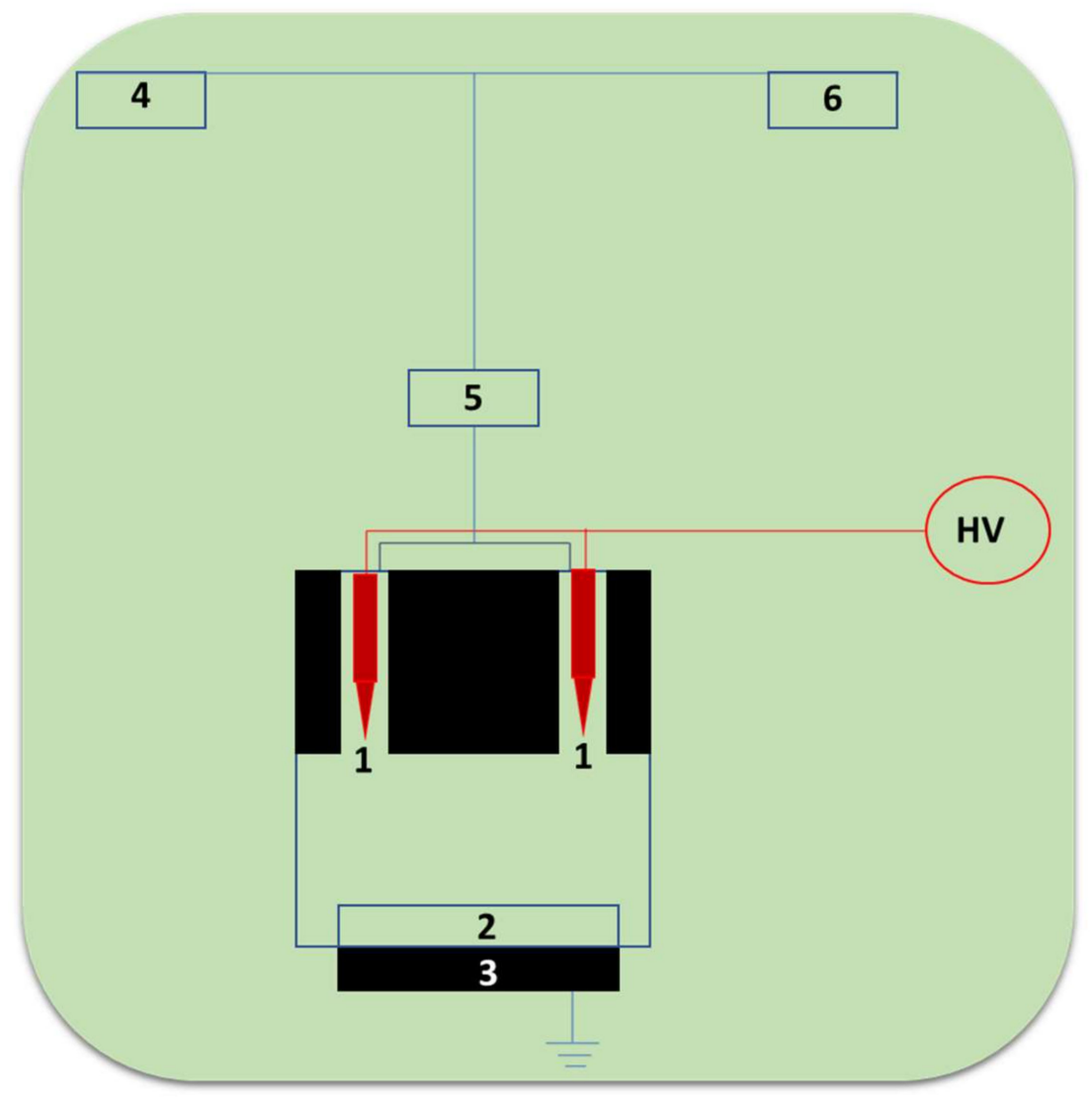

Figure 8. The schematic setup of the SPP apparatus for the biorecognition layer of laccase cross-linking inside of the plasma zone (1-pointed tungsten electrodes, 2-conductive solid support, 3-grounded electrode, 4-laccase enzyme solution, 5-atomizer, 6-helium gas). 
In addition to this, the Electrospray Ionization (ESI) technique allows us to bring in biomacromolecules such as proteins, enzymes, DNA strengths, oligonucleotides, or nanoparticles of molecular mass ( $\mathrm{kDa}$ to $\mathrm{MDa}$ ) range, as conformationally active and isolated units in the gas phase at ambient pressure conditions [83]. Castrovilli et al., 2020 explored the option to use Electrospray Ionization (ESI), a soft ionization technique, to deposit laccase over the electrode surface and to fabricate competitive biosensors for detection of the catechol [83]. The typical structure of the Electrospray Ionization (ESI) method for laccase-based biosensor development and the subsequent application of catechol detection is shown in Figure 9. However, a close examination revealed that the use of the solvent in this method, and its effect on the laccase confirmational/structural integrity, need to be overcome. Thus, Castrovilli et al., 2020 developed a unique laccase-solvent (methanol) combination to confirm the excellent laccase-based biocatalysis for catechol detection. By and large, this report [83] has a huge significance in laccase- or other enzyme-based biosensors, as it allows for a direct loading of the laccase/other enzymes over the electrode surface.

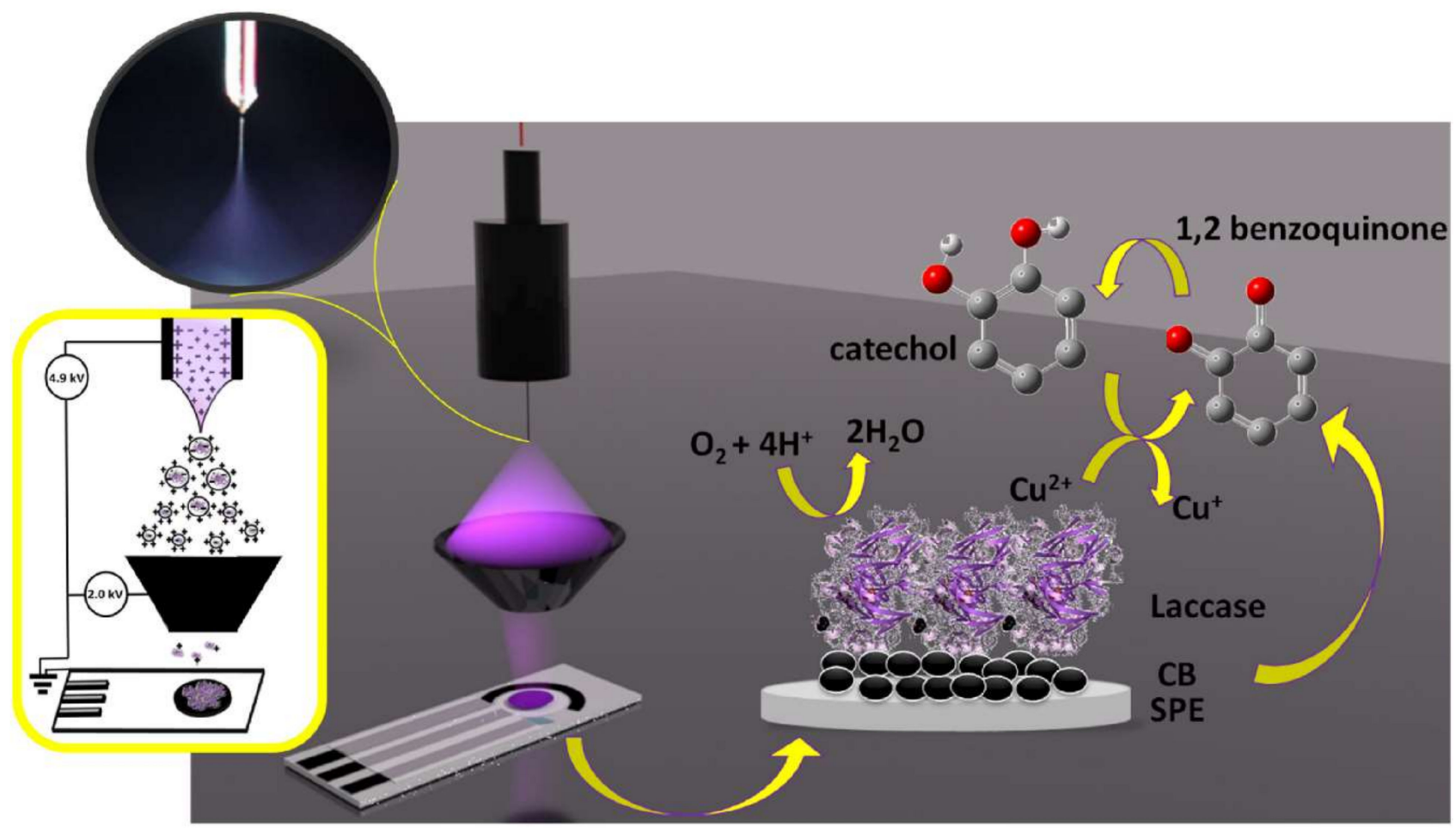

Figure 9. The schematic presentation of the application of ESI-based laccase biosensor development. This figure and its caption were adapted from Castrovilli et al., 2020 [83] with permission from Elsevier, Amsterdam, The Netherlands (License number 5218690964733). On the left side, the electrospray deposition (ESD) of the setup used for laccase immobilization on carbon black modified screen-printed electrodes; in the inset, a picture of the Taylor cone recorded by the camera during a deposition run. On the right side, the laccase catalytic action to catechol oxidation and oxygen reduction is underlined by the arrows.

Both the SPP and EI techniques (Figures 8 and 9) enable laccase to self-polymerize and successfully loaded the electrode surface with an active and functional laccase layer. This is extremely advantageous, considering the development of third-generation laccase biosensors. This laccase cross-linking technique is very important and holds a huge potential for the development of the biosensor field, through the direct incorporation of the cross-linked laccase onto the polymers/electropolymers/conductive nanomaterials. This will enable an easier construction of laccase-based biosensors. The analytical performance parameters of the SPP devices suggest that they can simplify biosensor production by enabling a faster availability and lower production costs. Thus, SPP, an entrapment biomolecule immobiliza- 
tion technique, needs to be examined in more detail, as it can offer huge possibilities to the biosensor field.

Moreover, the immobilization of laccase via cross-linked enzyme aggregates (CLEA) holds huge possibilities for the biosensor field, as these aggregates can efficaciously catalyze the oxidation of phenolic compounds with multilayer laccase loading. This will also reduce the cost of biosensor development. There are very few reports available for the laccase cross-linked aggregates in biosensor applications. Researchers in the biosensor field should therefore explore the development of significant biosensors employing this CLEA technology $[84,85]$.

\subsection{Covalent-Based Laccase Immobilization}

In the covalent-based immobilizations of laccase biosensors, the laccase is immobilized over the nanostructure-modified surface of the electrode (glassy carbon electrode or screenprinted carbon electrode) via covalent linkage. The covalent linkage was achieved mostly via the suitable cross-linking agent [26]. The cross-linking agents provide bi-functional groups: on one side, the enzyme can be immobilized, while the other functional group involves the covalent linkage with the electrode surface [46]. Hence, cross-linking agents play a vital role in covalent laccase immobilization protocols. In the typical reaction between glutaraldehyde and laccase/nano-supports, one of the aldehydic groups reacts with an amino group of the amino acid (lysine) of the enzyme/nano-supports to form the covalent linkage [47]. Most of the time the cross-linking agent glutaraldehyde is used in the covalent type of immobilization used in biosensors (Table 1). Cevher et al., 2021 reported the immobilization of the laccase on a PM1 electropolymer surface by covalent linkage via glutaraldehyde [66]. This biosensor was successfully applied for the stable detection of the indenoquinoxalinone from water. Similarly, glutaraldehyde-mediated laccase immobilized on electrodes of biosensors was reported by several researchers $[68,69,72-76,78]$. The typical glutaraldehyde-mediated immobilization of laccase in the laccase biosensor is shown in Figure 10 [69]. Sangubotla et al., 2021 synthesized the curcumin- and dimethylformamidemodified carbon dots (CDD-CDs) [70]. These CDD-CDs were first functionalized by APT (3-aminopropyltriethoxysilane), and then by APT-CDs linked to glutaraldehyde. Further, laccase was covalently immobilized on the APT-CDs (Figure 10). This optical laccase biosensor system provided an excellent detection of dopamine in human serum and cerebrospinal fluid. This biosensor exhibited a significant pharmacological research potential.

In addition, Sangubotla and kim, 2021 compared the physical adsorption-based immobilization and the covalent-based immobilization of laccase on the developed nano-support of CDD-CDs [69]. The obtained results were very convincing for covalent linkages of laccase over CDD-CDs. They indicated that the covalent form of the linkage produced detectable photoluminescence quenching for dopamine; however, the physical adsorption of laccase does not show the photoluminescence quenching. Thus, Sangubotla and Kim, 2021 confirmed the importance of the chemical (covalent) linkage form of immobilization over the physical adsorption [69]. To achieve covalent immobilization, it is important to have suitable chemically modifiable groups over the surface of the nano-support. Most of the time these groups include carboxyl, amine, hydroxyl, and aldehyde. The incorporation of these groups over the electrode surfaces can be achieved through various techniques, such as plasma polymerization, electrochemical, dipping, spin-coating, self-assembled mono-layer deposition, etc. [38].

Furthermore, another covalent immobilization strategy widely used in many laccasebased biosensors is the EDC/NHS (1-ethyl-3-(3-dimethylaminopropyl)carbodiimide/Nhydroxysuccinimide) activation reaction $[67,68,70,77]$. We have schematically drawn the typical EDC/NHS immobilization strategy to inform readers (Figure 11). In the chemistry of EDC/NHS activation, the carboxyl group of the nanostructured materials on the electrode surface was activated by the EDC/NHS, which forms a typical NHS-ester; further, the amino group from the laccase acts on this NHS-ester and forms the covalent linkage. This covalent linkage is very strong and provides additional stability to the immobilized laccase 
biocatalysis. In addition, this strategy can also be applied in reverse form; in this case, the carboxyl group of the laccase can be activated by the EDC/NHS strategy, and the amino groups of the modified nanostructured electrode were used for the formation of the covalent immobilization of laccase [38]. Either way, the formation of the covalent bond gives laccase additional stability for biocatalysis and subsequently enhances the sensor performance. Thus, from the biocatalysis and stability perspective of the immobilized laccase, immobilization via covalent bonding seems to be the best means of enzyme attachment [38]. The most predominant covalent immobilization strategies used are glutaraldehyde-based and EDC/NHS-based for laccase biosensors, according to the recent literature survey. Hence, there is a huge potential for employing other covalent immobilization strategies [86], for laccase-based immobilization, and enhancing the efficacy of the laccase-based biosensors.

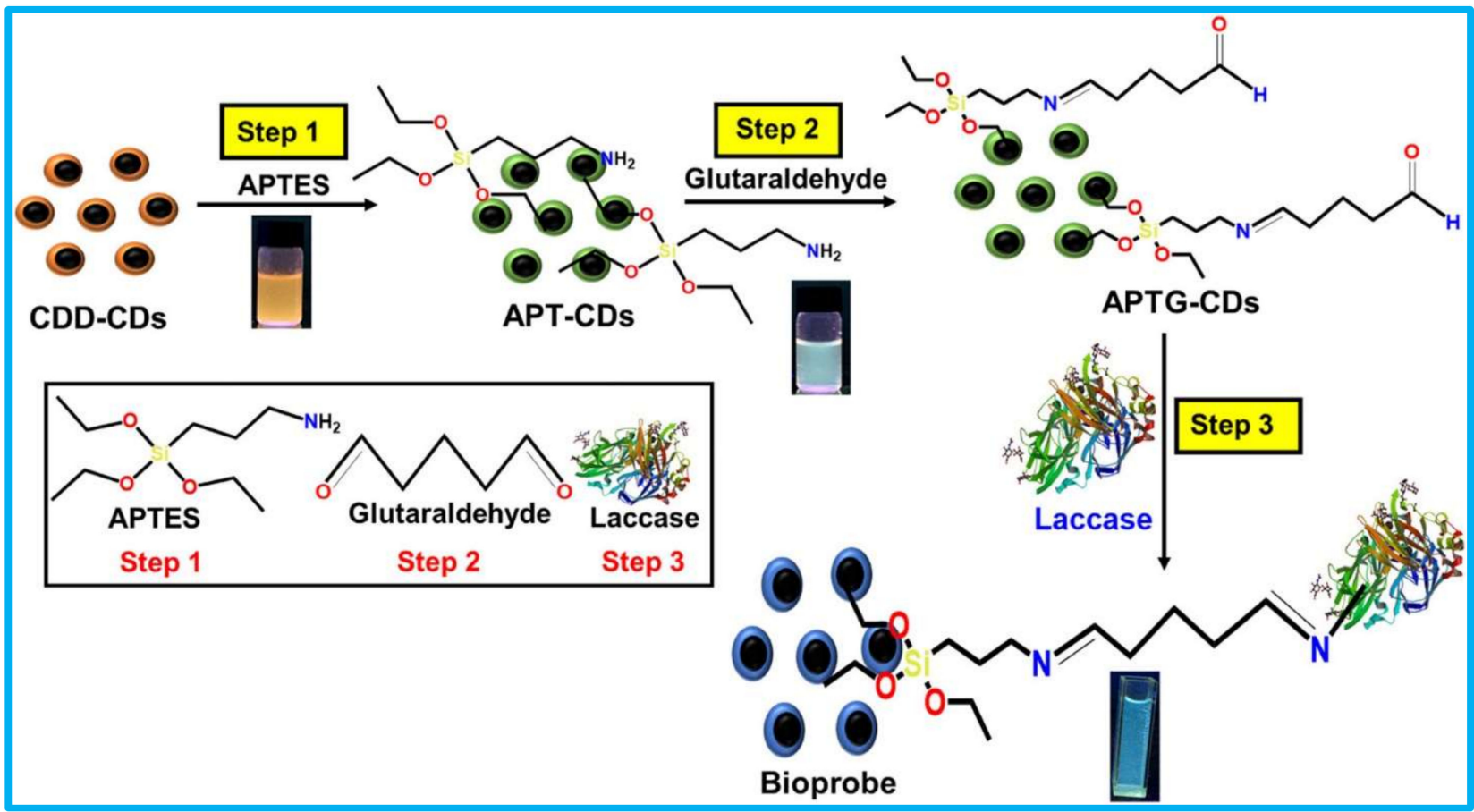

Figure 10. Schematic design for the silica-functionalization of CDD-CDs (curcumin- and dimethylformamide-modified carbon dots) and the laccase covalent immobilization on CDD-CDs via glutaraldehyde. (This image is adapted from the Sangubotla and Kim, 2021 [69] with permission from Elsevier, Amsterdam, The Netherlands (License number 5217721209232).

These covalent bindings caused partial conformational changes in the laccase, with a subsequent loss of activity [87]. An alteration in the substrate specificity of laccase after immobilization was reported by Jabbari and Khajeh et al., 2015 [87]. They investigated the SPR response, HPLC, and the intrinsic fluorescence studies and concluded that 'covalent immobilization alters the laccase response (due to confirmational changes) towards its substrate'. In their study, the free laccase oxidized the substrate ABTS easily; however, in its covalently immobilized form, laccase was unable to form the required confirmation (due to the multipoint attachment) to oxidize the substrate ABTS. This study has demonstrated that the activity of laccase toward its conventional substrates was affected by covalent coupling immobilization onto the CMD chip surface [87]. Furthermore, other studies also confirmed the substrate specificity change or confirmational change after the covalent immobilization process [88-90].

Thus, among the major advantages of the covalent technique, the obtained structural alterations or varied substrate specificities in laccase can be manipulated to obtain favorable effects as an improved specificity toward the specific phenolic substrate. As a result, different kinds of covalent immobilization approaches can alter the response of laccase to 
the phenolic substrates. This can be utilized for the development of more diverse laccasebased biosensors. In addition, covalent immobilization ensures the permeant binding of laccase via a covalent bond. This maintains the content laccase concentration in the biosensor. Consequently, it enhances the repeatability of the developed laccase biosensor. The major limitation of covalent immobilization is the fact that it is a multi-step, tedious, and laborious process. After all, our assessment of the covalent immobilization in this review suggests that the application of different available covalent immobilization approaches could lead to highly advanced and stable laccase biosensors.

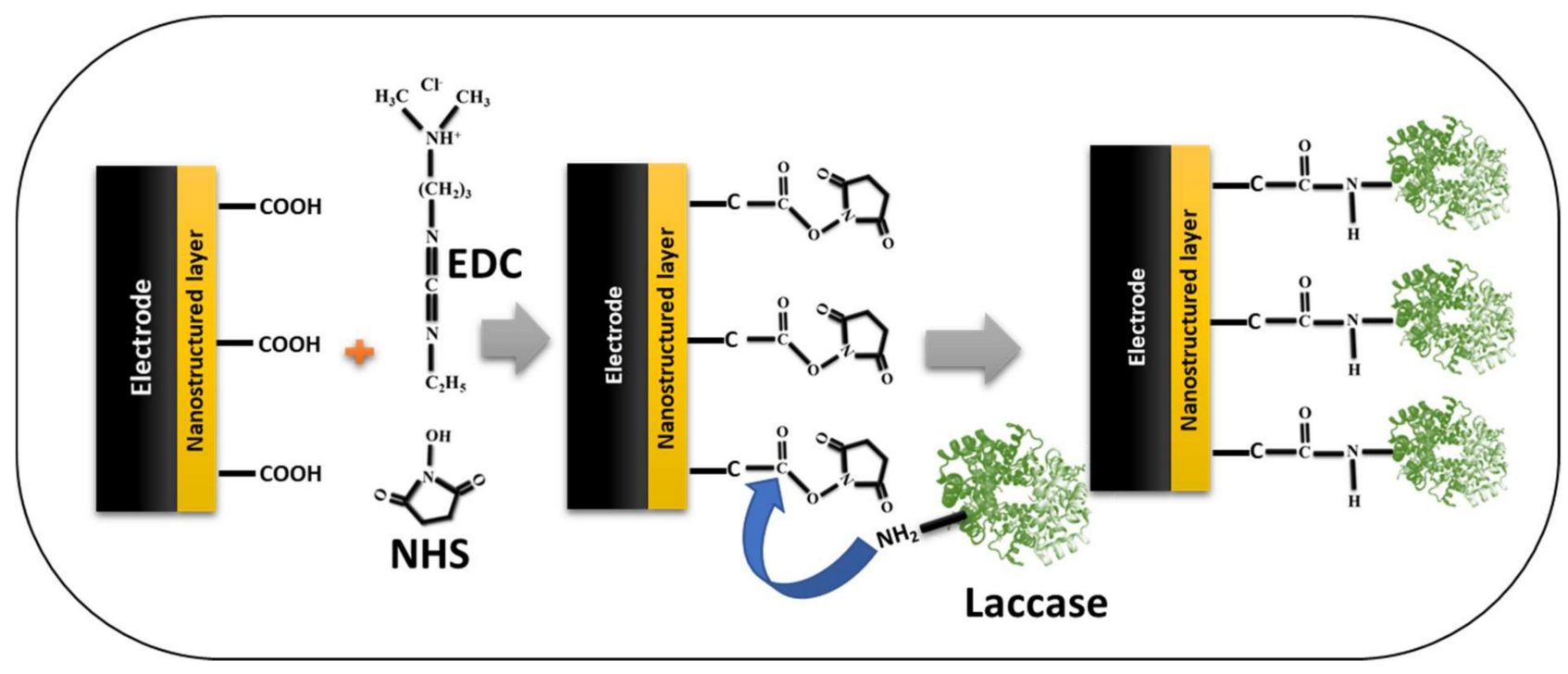

Figure 11. Schematic presentation of the EDC/NHS-based strategy of covalent immobilization of the laccase on the nanostructured-electrode/sensor platform in laccase-based biosensors.

\section{Conclusions and Future Perspectives}

The extensive recent literature assessment we made for this review article suggested that laccase-based biosensors are rapidly evolving. Novel laccase immobilization methodologies were applied and confronted in increasingly challenging and practical circumstances, overcoming the previous limitations of reliability and sensibility of the laccase biosensors. These upcoming immobilization technologies challenge the former limitations in connection with the efficacy and trustworthiness of their application. The technoeconomic aspects of all accessed nano-immobilization carriers in this review suggests that the adsorption and entrapment techniques are economically feasible, as they involve easy and simple process/methodologies, while the other methods, such as cross-linking, have high-energy and high-pressure requirements, covalent coupling needs, and more controlled protocols. Though these methods are less economically feasible than adsorption and entrapment, they possess extremely high stabilities, a better sensing performance, and multiple cycle applicability. The most widely used nanoscale nature of the immobilization carriers increases the cost of biosensors compared to the conventional carriers. Nevertheless, with nanoscale-based carriers, many researchers achieved new significant heights in terms of stable, rapid, ultra-sensitive, and multi-functional laccase biosensor response. In addition to the immobilization methods/immobilization carriers, the techno-economic aspect of laccase-based biosensors also depends upon the selection of the sensing platforms, the source of the laccase used, and the mode of the target biosensor application.

Our review revealed that the entrapment of laccase molecules on the biosensor electrode surface is the most used immobilization technique to date. Many previous reports suggest that conventional entrapment techniques have the constraint of the organo-polymeric matrices' interference with the laccase biosensing response. This limitation was meticulously tackled in recent times by many researchers, with the development of electrocon- 
ductive polymeric matrices. Thus, more focused entrapment technologies are coming up, with electro-conductive polymers or matrices which tend to interfere less with biosensing outputs. In this review, we found that, in addition to the electrical conductivity of the support materials, the analytical performance of laccase-based biosensors was also significantly improved due to the larger surface area for anchoring laccase in the nanostructured materials. In electrochemical transduction, we found that amperometry plays a major role, followed by potentiometric techniques and voltammetric techniques.

Our review reveals that the newly introduced biomineralization technique of the entrapment of laccase holds a huge potential, as it locks the functionally active laccase molecule in an inorganic three-dimensional nanomicrostructure. This allows for the conduction of the electron with functionally active laccase. In the future, more such biomineralization-based laccase entrapment technologies will significantly improve the laccase-based biosensors for phenolic compound detection.

This review predominantly conveys the importance of increasing the functionally active layers of laccase over the nanostructured electrode surface to stimulate the biosensor performance. The less functionally active laccase layer over the nanostructured electrode is the major lacking point found in most of the immobilization techniques in laccase biosensors. This can be further improved by employing the utilization of laccase cross-linking technologies such as cross-linked enzyme aggregates (CLEA) over the electrode surfaces. Very few reports were available in this regard, and this technique is very important for future laccase-based biosensors. The laccase cross-linking achieved via the Soft Plasma Polymerization (Figure 8) and Electrospray Ionization (ESI) (Figure 9) techniques holds great promise, as it directly loads the laccase layer over the electrode surface. The method to deposit laccase through this kind of automated procedure has the strong advantage of achieving an instrumental control over the quantity of laccase deposited on the nanostructured electrode surface, along with a better productivity of the biosensor. Until now, very few attempts were made in this regard. In our perspective, this might be due to the requirement of high energies and energy fluxes. In covalent-based laccase immobilization, it is highly important to note that covalent bonding affects the structural integrity (laccase confirmation); but, on the other hand, this attachment changes the substrate's specificity with regard to the phenolic compounds. Thus, the application of more and more covalent-based laccase immobilization tactics will increase the diversity of the laccase-based biosensors. In addition, exploring the nanostructures mimicking laccase catalysis (nanozyme [91]) for laccase immobilization will yield highly beneficial laccase-nanozyme-based biosensors. This nanozyme-coupled laccase catalysis may pave the way for the fabrication of third-generation laccase biosensors.

Thus, in this review, we examined all conventional, recent/advanced, and upcoming immobilization approaches in laccase-based biosensors. We believe that these novel improvements in nano-immobilization support for laccase-based biosensors will be implemented in the coming years, to detect the diversity of phenolic compounds in many fields, such as environment, food, and pharmacological research.

Author Contributions: Conceptualization, A.A.K. and J.-S.S.; methodology, A.A.K., J.-S.S., R.G.S., G.S.G., M.K., A.S., V.K.M., R.R.P. and G.D.S.; validation, A.A.K. and J.-S.S.; formal analysis, A.A.K. and J.-S.S.; investigation, A.A.K. and J.-S.S.; resources, A.A.K. and J.-S.S.; data curation, A.A.K.; writing —original draft preparation, A.A.K. and J.S.S.; writing—review and editing, A.A.K., J.S.S., R.G.S., G.S.G., M.K., A.S., V.K.M., R.R.P. and G.D.S.; supervision, A.A.K. and J.-S.S.; project administration A.A.K. and J.-S.S.; funding acquisition, A.A.K. and J.-S.S. All authors have read and agreed to the published version of the manuscript.

Funding: This work was supported by the National Research Foundation of Korea (NRF), through a grant funded by the Korean government (MSIT) (NRF-2019R1G1A1009363).

Institutional Review Board Statement: Not applicable.

Informed Consent Statement: Not applicable. 
Data Availability Statement: Not applicable.

Acknowledgments: This work was supported by the Dongguk University-Seoul, South Korea, under Research Fund 2021-2023.

Conflicts of Interest: The authors declare that they have no conflict of interest.

\section{References}

1. Bilal, M.; Rasheed, T.; Nabeel, F.; Iqbal, H.M.N.; Zhao, Y. Hazardous contaminants in the environment and their laccase-assisted degradation-A review. J. Environ. Manag. 2019, 234, 253-264. [CrossRef]

2. Anku, W.W.; Mamo, M.A.; Mamo, M.A.; Penny, P.W.; Govender, P.P. Phenolic Compounds Compounds in Water: Sources, Phenolic Toxicity and Treatment Methods. In Phenolic Compounds: Natural Sources, Importance and Applications; Intech Open: London, UK, 2017; pp. 419-443.

3. Barrico, M.L.; Nabais, C.; Martins, M.J.; Freitas, H. Sources of phenolic compounds in two catchments of southern Portugal-Effect of season, land use and soil type. Chemosphere 2006, 65, 482-488. [CrossRef]

4. Stadlmair, L.F.; Letzel, T.; Graßmann, J. Monitoring enzymatic degradation of emerging contaminants using a chip-based robotic nano-ESI-MS tool. Anal. Bioanal. Chem. 2018, 410, 27-32. [CrossRef]

5. Yuan, T.; Tazaki, A.; Hashimoto, K.; Al Hossain, M.M.A.; Kurniasari, F.; Ohgami, N.; Aoki, M.; Ahsan, N.; Akhand, A.A.; Kato, M. Development of an efficient remediation system with a low cost after identification of water pollutants including phenolic compounds in a tannery built-up area in Bangladesh. Chemosphere 2021, 280, 130959. [CrossRef] [PubMed]

6. Acosta, C.A.; Pasquali, C.E.L.; Paniagua, G.; Garcinuño, R.M.; Hernando, P.F. Evaluation of total phenol pollution in water of San Martin Canal from Santiago del Estero, Argentina. Environ. Pollut. 2018, 236, 265-272. [CrossRef] [PubMed]

7. $\quad$ Parada, H.; Gammon, M.D.; Ettore, H.L.; Chen, J.; Calafat, A.M.; Neugut, A.I.; Santella, R.M.; Wolff, M.S.; Teitelbaum, S.L. Urinary concentrations of environmental phenols and their associations with breast cancer incidence and mortality following breast cancer. Environ. Int. 2019, 130, 104890. [CrossRef] [PubMed]

8. Agency for Toxic Substances and Disease Registry. Toxicological Profile for Phenol; U.S. Department of Health and Human Services, Public Health Services: Atlanta, GA, USA, 2008.

9. Rodríguez-Delgado, M.M.; Alemán-Nava, G.S.; Rodríguez-Delgado, J.M.; Dieck-Assad, G.; Martínez-Chapa, S.O.; Barceló, D.; Parra, R. Laccase-based biosensors for detection of phenolic compounds. TrAC-Trends Anal. Chem. 2015, 74, 21-45. [CrossRef]

10. Delfino, I.; Diano, N.; Lepore, M. Advanced optical sensing of phenolic compounds for environmental applications. Sensors 2021, 21, 7563. [CrossRef]

11. Karim, F.; Fakhruddin, A.N.M. Recent advances in the development of biosensor for phenol: A review. Rev. Environ. Sci. Biotechnol. 2012, 11, 261-274. [CrossRef]

12. Pasternak, G.; Hanczyc, M.M. Novel method for detecting and quantifying phenol with transient response of glycolytic oscillations of synchronised yeast cells. Sens. Bio-Sens. Res. 2019, 22, 100259. [CrossRef]

13. Flachbart, L.K.; Gerhard, C.; Gertzen, W.; Gohlke, H.; Marienhagen, J. Development of a Biosensor Platform for Phenolic Compounds Using a Transition Ligand Strategy. ACS Synth. Biol. 2021, 10, 2002-2014. [CrossRef] [PubMed]

14. Sohrabi, H.; Hemmati, A.; Majidi, M.R.; Eyvazi, S.; Jahanban-Esfahlan, A.; Baradaran, B.; Adlpour-Azar, R.; Mokhtarzadeh, A.; de la Guardia, M. Recent advances on portable sensing and biosensing assays applied for detection of main chemical and biological pollutant agents in water samples: A critical review. TrAC-Trends Anal. Chem. 2021, 143, 116344. [CrossRef]

15. Forzato, C.; Vida, V.; Berti, F. Biosensors and Sensing Systems for Rapid Analysis of Phenolic Compounds from Plants: A Comprehensive Review. Biosensors 2020, 10, 105. [CrossRef] [PubMed]

16. Perna, V.; Meyer, A.S.; Holck, J.; Eltis, L.D.; Eijsink, V.G.H.; Wittrup Agger, J. Laccase-Catalyzed Oxidation of Lignin Induces Production of $\mathrm{H}_{2} \mathrm{O}_{2}$. ACS Sustain. Chem. Eng. 2020, 8, 831-841. [CrossRef]

17. Mehra, R.; Muschiol, J.; Meyer, A.S.; Kepp, K.P. A structural-chemical explanation of fungal laccase activity. Sci. Rep. 2018, 8, 1-16. [CrossRef] [PubMed]

18. Xu, F.; Kulys, J.J.; Duke, K.; Li, K.; Krikstopaitis, K.; Deussen, H.J.W.; Abbate, E.; Galinyte, V.; Schneider, P. Redox chemistry in laccase-catalyzed oxidation of N-hydroxy compounds. Appl. Environ. Microbiol. 2000, 66, 2052-2056. [CrossRef]

19. Mazlan, S.Z.; Lee, Y.H.; Hanifah, S.A. A new Laccase based biosensor for tartrazine. Sensors 2017, 17, 2859. [CrossRef]

20. Mei, L.P.; Feng, J.J.; Wu, L.; Zhou, J.Y.; Chen, J.R.; Wang, A.J. Novel phenol biosensor based on laccase immobilized on reduced graphene oxide supported palladium-copper alloyed nanocages. Biosens. Bioelectron. 2015, 74, 347-352. [CrossRef]

21. Vasilescu, I.; Eremia, S.A.V.; Kusko, M.; Radoi, A.; Vasile, E.; Radu, G.L. Molybdenum disulphide and graphene quantum dots as electrode modifiers for laccase biosensor. Biosens. Bioelectron. 2016, 75, 232-237. [CrossRef]

22. Wang, X.; Liu, J.; Qu, R.; Wang, Z.; Huang, Q. The laccase-like reactivity of manganese oxide nanomaterials for pollutant conversion: Rate analysis and cyclic voltammetry. Sci. Rep. 2017, 7, 1-10. [CrossRef]

23. Parra Guardado, A.L.; Belleville, M.P.; Rostro Alanis, M.D.J.; Parra Saldivar, R.; Sanchez-Marcano, J. Effect of redox mediators in pharmaceuticals degradation by laccase: A comparative study. Process Biochem. 2019, 78, 123-131. [CrossRef]

24. Jeon, J.R.; Baldrian, P.; Murugesan, K.; Chang, Y.S. Laccase-catalysed oxidations of naturally occurring phenols: From in vivo biosynthetic pathways to green synthetic applications. Microb. Biotechnol. 2012, 5, 318-332. [CrossRef] 
25. Witayakran, S.; Ragauskas, A.J. Synthetic applications of laccase in green chemistry. Adv. Synth. Catal. 2009, 351, 1187-1209. [CrossRef]

26. Ghodake, G.S.; Yang, J.; Shinde, S.S.; Mistry, B.M.; Kim, D.Y.; Sung, J.S.; Kadam, A.A. Paper waste extracted A-cellulose fibers super-magnetized and chitosan-functionalized for covalent laccase immobilization. Bioresour. Technol. 2018, 261, 420-427. [CrossRef]

27. Martinez-Ortiz, J.; Flores, R.; Vazquez-Duhalt, R. Molecular design of laccase cathode for direct electron transfer in a biofuel cell. Biosens. Bioelectron. 2011, 26, 2626-2631. [CrossRef]

28. Agrawal, K.; Chaturvedi, V.; Verma, P. Fungal laccase discovered but yet undiscovered. Bioresour. Bioprocess. $2018,5,1-2$. [CrossRef]

29. Malhotra, M.; Suman, S.K. Laccase-mediated delignification and detoxification of lignocellulosic biomass: Removing obstacles in energy generation. Environ. Sci. Pollut. Res. 2021, 28, 58929-58944. [CrossRef]

30. Lade, H.; Kadam, A.; Paul, D.; Govindwar, S. A low-cost wheat bran medium for biodegradation of the benzidine-based carcinogenic dye trypan blue using a microbial consortium. Int. J. Environ. Res. Public Health 2015, 12, 3480. [CrossRef] [PubMed]

31. Arregui, L.; Ayala, M.; Gómez-Gil, X.; Gutiérrez-Soto, G.; Hernández-Luna, C.E.; Herrera De Los Santos, M.; Levin, L.; Rojo-Domínguez, A.; Romero-Martínez, D.; Saparrat, M.C.N.; et al. Laccases: Structure, function, and potential application in water bioremediation. Microb. Cell Fact. 2019, 18, 1-33. [CrossRef]

32. Kumar Mishra, S.; Kumar Srivastava, S.; Ash, K. Laccase sources and their applications in environmental pollution. Int. J. Life-Sci. Sci. Res. 2015, 1, 71-73.

33. Leontievsky, A.A.; Vares, T.; Lankinen, P.; Shergill, J.K.; Pozdnyakova, N.N.; Myasoedova, N.M.; Kalkkinen, N.; Golovleva, L.A.; Cammack, R.; Thurston, C.F.; et al. Blue and yellow laccases of ligninolytic fungi. FEMS Microbiol. Lett. 1997, 156, 9-14. [CrossRef]

34. Shraddha; Shekher, R.; Sehgal, S.; Kamthania, M.; Kumar, A. Laccase: Microbial sources, production, purification, and potential biotechnological applications. Enzym. Res. 2011, 2011, 217861. [CrossRef] [PubMed]

35. Chuang, E.Y.; Lin, P.Y.; Wang, P.F.; Kuo, T.R.; Chen, C.H.; Manga, Y.B.; Hsiao, Y.C. Label-free, smartphone-based, and sensitive nano-structural liquid crystal aligned by ceramic silicon compound-constructed dmoap-based biosensor for the detection of urine albumin. Int. J. Nanomed. 2021, 16, 763-773. [CrossRef] [PubMed]

36. Chen, F.-L.; Fan, Y.-J.; Lin, J.-D.; Hsiao, Y.-C. Label-free, color-indicating, and sensitive biosensors of cholesteric liquid crystals on a single vertically aligned substrate. Biomed. Opt. Express 2019, 10, 4636. [CrossRef] [PubMed]

37. Fan, Y.J.; Chen, F.L.; Liou, J.C.; Huang, Y.W.; Chen, C.H.; Hong, Z.Y.; De Lin, J.; Hsiao, Y.C. Label-free multi-microfluidic immunoassays with liquid crystals on polydimethylsiloxane biosensing chips. Polymers 2020, 12, 395. [CrossRef]

38. Ardhaoui, M.; Bhatt, S.; Zheng, M.; Dowling, D.; Jolivalt, C.; Khonsari, F.A. Biosensor based on laccase immobilized on plasma polymerized allylamine/carbon electrode. Mater. Sci. Eng. C 2013, 33, 3197-3205. [CrossRef] [PubMed]

39. Gkantzou, E.; Chatzikonstantinou, A.V.; Fotiadou, R.; Giannakopoulou, A.; Patila, M.; Stamatis, H. Trends in the development of innovative nanobiocatalysts and their application in biocatalytic transformations. Biotechnol. Adv. 2021, 51, 107738. [CrossRef] [PubMed]

40. Bai, X.; Gu, H.; Chen, W.; Shi, H.; Yang, B.; Huang, X.; Zhang, Q. Immobilized laccase on activated poly(vinyl alcohol) microspheres for enzyme thermistor application. Appl. Biochem. Biotechnol. 2014, 173, 1097-1107. [CrossRef] [PubMed]

41. Castrovilli, M.C.; Bolognesi, P.; Chiarinelli, J.; Avaldi, L.; Calandra, P.; Antonacci, A.; Scognamiglio, V. The convergence of forefront technologies in the design of laccase-based biosensors-An update. TrAC-Trends Anal. Chem. 2019, 119, 115615. [CrossRef]

42. Datta, S.; Veena, R.; Samuel, M.S.; Selvarajan, E. Immobilization of laccases and applications for the detection and remediation of pollutants: A review. Environ. Chem. Lett. 2021, 19, 521-538. [CrossRef]

43. Ansari, S.A.; Husain, Q. Potential applications of enzymes immobilized on/in nano materials: A review. Biotechnol. Adv. 2012, 30, 512-523. [CrossRef] [PubMed]

44. Li, D.; Luo, L.; Pang, Z.; Ding, L.; Wang, Q.; Ke, H.; Huang, F.; Wei, Q. Novel phenolic biosensor based on a magnetic polydopamine-laccase-nickel nanoparticle loaded carbon nanofiber composite. ACS Appl. Mater. Interfaces 2014, 6, 5144-5151. [CrossRef]

45. Kadam, A.A.; Sharma, B.; Shinde, S.K.; Ghodake, G.S.; Saratale, G.D.; Saratale, R.G.; Kim, D.Y.; Sung, J.S. Thiolation of chitosan loaded over super-magnetic halloysite nanotubes for enhanced laccase immobilization. Nanomaterials 2020, 10, 2560. [CrossRef]

46. Kadam, A.A.; Jang, J.; Jee, S.C.; Sung, J.S.; Lee, D.S. Chitosan-functionalized supermagnetic halloysite nanotubes for covalent laccase immobilization. Carbohydr. Polym. 2018, 194, 208-216. [CrossRef]

47. Kadam, A.A.; Jang, J.; Lee, D.S. Supermagnetically Tuned Halloysite Nanotubes Functionalized with Aminosilane for Covalent Laccase Immobilization. ACS Appl. Mater. Interfaces 2017, 9, 15492-15501. [CrossRef]

48. Kim, M.; Jee, S.C.; Sung, J.S.; Kadam, A.A. Supermagnetic sugarcane bagasse hydrochar for enhanced osteoconduction in human adipose tissue-derived mesenchymal stem cells. Nanomaterials 2020, 10, 1793. [CrossRef]

49. Min, K.; Jee, S.C.; Sung, J.S.; Kadam, A.A. Anti-proliferative applications of laccase immobilized on super-magnetic chitosanfunctionalized halloysite nanotubes. Int. J. Biol. Macromol. 2018, 118, 228-237.

50. Ghodake, G.S.; Shinde, S.K.; Saratale, G.D.; Saratale, R.G.; Kim, M.; Jee, S.C.; Kim, D.Y.; Sung, J.S.; Kadam, A.A. $\alpha$-cellulose fibers of paper-waste origin surface-modified with $\mathrm{Fe}_{3} \mathrm{O}_{4}$ and thiolated-chitosan for efficacious immobilization of laccase. Polymers 2021, 13, 581. [CrossRef] 
51. Othman, A.M.; Wollenberger, U. Amperometric biosensor based on coupling aminated laccase to functionalized carbon nanotubes for phenolics detection. Int. J. Biol. Macromol. 2020, 153, 855-864. [CrossRef]

52. Wang, K.; Liu, P.; Ye, Y.; Li, J.; Zhao, W.; Huang, X. Fabrication of a novel laccase biosensor based on silica nanoparticles modified with phytic acid for sensitive detection of dopamine. Sens. Actuators B Chem. 2014, 197, 292-299. [CrossRef]

53. Palanisamy, S.; Ramaraj, S.K.; Chen, S.M.; Yang, T.C.K.; Pan, Y.F.; Chen, T.W.; Velusamy, V.; Selvam, S. A novel Laccase biosensor based on laccase immobilized graphene-cellulose microfiber composite modified screen-printed carbon electrode for sensitive determination of catechol. Sci. Rep. 2017, 7, 1-12. [CrossRef] [PubMed]

54. Zhang, M.; Zhang, Y.; Yang, C.; Ma, C.; Zhang, Y.; Tang, J. Synthesis of three-dimensional laccase-Cu $\mathrm{Cu}_{3}\left(\mathrm{PO}_{4}\right)_{2} \cdot 3 \mathrm{H}_{2} \mathrm{O}$ microflowers via biomineralization for UV-vis epinephrine biosensing. Microchem. J. 2022, 172, 106911. [CrossRef]

55. Bravo, I.; Prata, M.; Torrinha, Á.; Delerue-Matos, C.; Lorenzo, E.; Morais, S. Laccase bioconjugate and multi-walled carbon nanotubes-based biosensor for bisphenol A analysis. Bioelectrochemistry 2021, 144, 108033. [CrossRef] [PubMed]

56. Zhang, M.; Zhang, Y.; Yang, C.; Ma, C.; Tang, J. A smartphone-assisted portable biosensor using laccase-mineral hybrid microflowers for colorimetric determination of epinephrine. Talanta 2021, 224, 121840. [CrossRef] [PubMed]

57. Mohtar, L.G.; Aranda, P.; Messina, G.A.; Nazareno, M.A.; Pereira, S.V.; Raba, J.; Bertolino, F.A. Amperometric biosensor based on laccase immobilized onto a nanostructured screen-printed electrode for determination of polyphenols in propolis. Microchem. $J$. 2019, 144, 13-18. [CrossRef]

58. Yang, J.; Li, D.; Fu, J.; Huang, F.; Wei, Q. TiO $2-\mathrm{CuCNF}_{\text {s }}$ based laccase biosensor for enhanced electrocatalysis in hydroquinone detection. J. Electroanal. Chem. 2016, 766, 16-23. [CrossRef]

59. Eremia, S.A.V.; Vasilescu, I.; Radoi, A.; Litescu, S.C.; Radu, G.L. Disposable biosensor based on platinum nanoparticles-reduced graphene oxide-laccase biocomposite for the determination of total polyphenolic content. Talanta 2013, 110, 164-170. [CrossRef]

60. Zhang, Y.; Li, X.; Li, D.; Wei, Q. A laccase based biosensor on AuNPs-MoS 2 modified glassy carbon electrode for catechol detection. Colloids Surf. B Biointerfaces 2020, 186, 110683. [CrossRef]

61. Rubio-Govea, R.; Hickey, D.P.; García-Morales, R.; Rodriguez-Delgado, M.; Domínguez-Rovira, M.A.; Minteer, S.D.; Ornelas-Soto, N.; García-García, A. $\mathrm{MoS}_{2}$ nanostructured materials for electrode modification in the development of a laccase based amperometric biosensor for non-invasive dopamine detection. Microchem. J. 2020, 155, 104792. [CrossRef]

62. Kavetskyy, T.; Smutok, O.; Demkiv, O.; Mat'ko, I.; Švajdlenková, H.; Šauša, O.; Novák, I.; Berek, D.; Čechová, K.; Pecz, M.; et al. Microporous carbon fibers as electroconductive immobilization matrixes: Effect of their structure on operational parameters of laccase-based amperometric biosensor. Mater. Sci. Eng. C 2020, 109, 110570. [CrossRef]

63. Chen, T.; Xu, Y.; Peng, Z.; Li, A.; Liu, J. Simultaneous Enhancement of Bioactivity and Stability of Laccase by Cu ${ }^{2+} /$ PAA/PPEGA Matrix for Efficient Biosensing and Recyclable Decontamination of Pyrocatechol. Anal. Chem. 2017, 89, 2065-2072. [CrossRef] [PubMed]

64. Coelho, J.H.; Eisele, A.P.P.; Valezi, C.F.; Mattos, G.J.; Schirmann, J.G.; Dekker, R.F.H.; Barbosa-Dekker, A.M.; Sartori, E.R. Exploring the exocellular fungal biopolymer botryosphaeran for laccase-biosensor architecture and application to determine dopamine and spironolactone. Talanta 2019, 204, 475-483. [CrossRef]

65. Wardak, C.; Paczosa-Bator, B.; Malinowski, S. Application of cold plasma corona discharge in preparation of laccase-based biosensors for dopamine determination. Mater. Sci. Eng. C 2020, 116, 111199. [CrossRef] [PubMed]

66. Cevher, S..C.; Bekmezci, S.A.; SaniyeSoylemez; Udum, Y.A.; Toppare, L.; Çırpan, A. Indenoquinoxalinone based conjugated polymer substrate for laccase biosensor. Mater. Chem. Phys. 2021, 257, 123788. [CrossRef]

67. Gomes, A.; Mattos, G.J.; Coldibeli, B.; Dekker, R.F.H.; Barbosa Dekker, A.M.; Sartori, E.R. Covalent attachment of laccase to carboxymethyl-botryosphaeran in aqueous solution for the construction of a voltammetric biosensor to quantify quercetin. Bioelectrochemistry 2020, 135, 107543. [CrossRef]

68. Zhao, K.; Veksha, A.; Ge, L.; Lisak, G. Near real-time analysis of para-cresol in wastewater with a laccase-carbon nanotube-based biosensor. Chemosphere 2021, 269, 128699. [CrossRef]

69. Sangubotla, R.; Kim, J. Fiber-optic biosensor based on the laccase immobilization on silica-functionalized fluorescent carbon dots for the detection of dopamine and multi-color imaging applications in neuroblastoma cells. Mater. Sci. Eng. C 2021, 122, 111916. [CrossRef]

70. Karami, C.; Taher, M.A. A catechol biosensor based on immobilizing laccase to $\mathrm{Fe}_{3} \mathrm{O}_{4} @ \mathrm{Au}$ core-shell nanoparticles. Int. J. Biol. Macromol. 2019, 129, 84-90. [CrossRef]

71. Patel, S.K.S.; Anwar, M.Z.; Kumar, A.; Otari, S.V.; Pagolu, R.T.; Kim, S.Y.; Kim, I.W.; Lee, J.K. Fe ${ }_{2} \mathrm{O}_{3}$ yolk-shell particle-based laccase biosensor for efficient detection of 2,6-dimethoxyphenol. Biochem. Eng. J. 2018, 132, 1-8. [CrossRef]

72. Romero-Arcos, M.; Garnica-Romo, M.G.; Martínez-Flores, H.E. Characterization of Amperometric Laccase Biosensor Based on Carbon Nanotube. Procedia Technol. 2017, 27, 279-281. [CrossRef]

73. Maleki, N.; Kashanian, S.; Nazari, M.; Shahabadi, N. A novel sensitive laccase biosensor using gold nanoparticles and poly L-arginine to detect catechol in natural water. Biotechnol. Appl. Biochem. 2019, 66, 502-509. [CrossRef] [PubMed]

74. Yasa, M.; Deniz, A.; Forough, M.; Yildirim, E.; Persil Cetinkol, O.; Udum, Y.A.; Toppare, L. Construction of amperometric biosensor modified with conducting polymer/carbon dots for the analysis of catechol. J. Polym. Sci. 2020, 58, 3336-3348. [CrossRef]

75. Chakroun Galai, H.; Rassas, I.; Namour, P.; Bonhomme, A.; Raimondi, G.; Besbes Hentati, S.; Jaffrezic-Renault, N. A Laccase/Chitosan-Lambda-Carrageenan Based Voltammetric Biosensor for Phenolic Compound Detection. Electroanalysis 2020, 32, 732-740. [CrossRef] 
76. Bounegru, A.V.; Apetrei, C. Development of a novel electrochemical biosensor based on carbon nanofibers-cobalt phthalocyaninelaccase for the detection of p-coumaric acid in phytoproducts. Int. J. Mol. Sci. 2021, 22, 9302. [CrossRef] [PubMed]

77. Bonet-San-emeterio, M.; Montiel, N.F.; Del Valle, M. Graphene for the building of electroanalytical enzyme-based biosensors. Application to the inhibitory detection of emerging pollutants. Nanomaterials 2021, 11, 2094. [CrossRef] [PubMed]

78. Salvo-Comino, C.; González-Gil, A.; Rodriguez-Valentin, J.; Garcia-Hernandez, C.; Martin-Pedrosa, F.; Garcia-Cabezon, C.; Rodriguez-Mendez, M.L. Biosensors platform based on chitosan/AuNPs/phthalocyanine composite films for the electrochemical detection of catechol. the role of the surface structure. Sensors 2020, 20, 2152. [CrossRef]

79. Mercante, L.A.; Pavinatto, A.; Pereira, T.S.; Migliorini, F.L.; dos Santos, D.M.; Correa, D.S. Nanofibers interfaces for biosensing: Design and applications. Sens. Actuators Rep. 2021, 3, 100048. [CrossRef]

80. Litescu, S.C.; Eremia, S.A.V.; Bertoli, A.; Pistelli, L.; Radu, G.L. Laccase-nafion based biosensor for the determination of polyphenolic secondary metabolites. Anal. Lett. 2010, 43, 1089-1099. [CrossRef]

81. Sigawi, S.; Smutok, O.; Demkiv, O.; Zakalska, O.; Gayda, G.; Nitzan, Y.; Nisnevitch, M.; Gonchar, M. Immobilized formaldehydemetabolizing enzymes from Hansenula polymorpha for removal and control of airborne formaldehyde. J. Biotechnol. 2011, 153, 138-144. [CrossRef] [PubMed]

82. Malinowski, S.; Wardak, C.; Jaroszyńska-Wolińska, J.; Herbert, P.A.F.; Panek, R. Cold Plasma as an Innovative Construction Method of Voltammetric Biosensor Based on Laccase. Sensors 2018, 18, 4086. [CrossRef]

83. Castrovilli, M.C.; Bolognesi, P.; Chiarinelli, J.; Avaldi, L.; Cartoni, A.; Calandra, P.; Tempesta, E.; Giardi, M.T.; Antonacci, A.; Arduini, F.; et al. Electrospray deposition as a smart technique for laccase immobilisation on carbon black-nanomodified screen-printed electrodes. Biosens. Bioelectron. 2020, 163, 112299. [CrossRef]

84. Sheldon, R.A. Cross-Linked Enzyme Aggregates as Industrial Biocatalysts. Pharm. Process Chem. 2010, 15, 159-181.

85. Hong, J.; Jung, D.; Park, S.; Oh, Y.; Oh, K.K.; Lee, S.H. Immobilization of laccase via cross-linked enzyme aggregates prepared using genipin as a natural cross-linker. Int. J. Biol. Macromol. 2021, 169, 541-550. [CrossRef] [PubMed]

86. Wong, L.S.; Khan, F.; Micklefield, J. Selective covalent protein immobilization: Strategies and applications. Chem. Rev. 2009, 109, 4025-4053. [CrossRef]

87. Jabbari, S.; Dabirmanesh, B.; Khajeh, K. Specificity enhancement towards phenolic substrate by immobilization of laccase on surface plasmon resonance sensor chip. J. Mol. Catal. B Enzym. 2015, 121, 32-36. [CrossRef]

88. Secundo, F. Conformational changes of enzymes upon immobilisation. Chem. Soc. Rev. 2013, 42, 6250-6261. [CrossRef]

89. Mateo, C.; Palomo, J.M.; Fernandez-Lorente, G.; Guisan, J.M.; Fernandez-Lafuente, R. Improvement of enzyme activity, stability and selectivity via immobilization techniques. Enzyme Microb. Technol. 2007, 40, 1451-1463. [CrossRef]

90. Rodrigues, R.C.; Ortiz, C.; Berenguer-Murcia, Á.; Torres, R.; Fernández-Lafuente, R. Modifying enzyme activity and selectivity by immobilization. Chem. Soc. Rev. 2013, 42, 6290-6307. [CrossRef]

91. Tran, T.D.; Nguyen, P.T.; Le, T.N.; Kim, M.I. DNA-copper hybrid nanoflowers as efficient laccase mimics for colorimetric detection of phenolic compounds in paper microfluidic devices. Biosens. Bioelectron. 2021, 182, 113187. [CrossRef] [PubMed] 